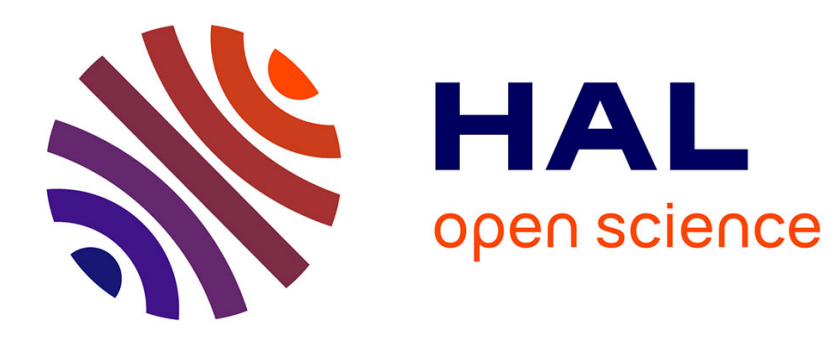

\title{
On p-tuples of the Grassmann manifolds
}

Joël Rouyer

\section{To cite this version:}

Joël Rouyer. On p-tuples of the Grassmann manifolds. 2010. hal-00491032

\section{HAL Id: hal-00491032 \\ https://hal.science/hal-00491032}

Preprint submitted on 10 Jun 2010

HAL is a multi-disciplinary open access archive for the deposit and dissemination of scientific research documents, whether they are published or not. The documents may come from teaching and research institutions in France or abroad, or from public or private research centers.
L'archive ouverte pluridisciplinaire HAL, est destinée au dépôt et à la diffusion de documents scientifiques de niveau recherche, publiés ou non, émanant des établissements d'enseignement et de recherche français ou étrangers, des laboratoires publics ou privés. 


\title{
On p-tuples of the Grassmann manifolds
}

\author{
Joël Rouyer
}

June 10, 2010

\begin{abstract}
We provide a matrix invarariant for isometry classes of $p$-tuples of points in the Grassmann manifold $G_{n}\left(\mathbb{K}^{d}\right)(\mathbb{K}=\mathbb{R}$ or $\mathbb{C})$. This invariant fully caracterizes the $p$-tuple. We use it to determine the regular $p$-tuples of $G_{2}\left(\mathbb{R}^{d}\right), G_{3}\left(\mathbb{R}^{d}\right)$ and $G_{2}\left(\mathbb{C}^{d}\right)$.
\end{abstract}

\section{Introduction and notation}

A triangle (triple of points) of the Euclidean space is fully defined, up to isometry, by three numbers, namely its side lengths. Of course, three given positive numbers may or may not be the side lengths of some triangle. Indeed, they are if and only if they satisfy the well known triangle inequality. More generally, we can consider $p$-tuples of points. Once again, a $p$-tuple of the Euclidean space is fully characterized by the data of the distances determined by each pair of points. The existence criterion is a little less easy, but could be stated in terms of signs of the minors of some matrix build up from the square of the prescribed distances (see for instance [1, p. 239]). The aim of this paper is too discuss analogous matter for $p$-tuples of points in the Grassmann manifold $G_{n}\left(\mathbb{K}^{p n}\right)$, where $\mathbb{K}=\mathbb{R}$ or $\mathbb{C}$. It will turn out that in this case, the data of distances between the points of a $p$-tuple are no longer sufficient to characterize it up to a global isometry of $\mathbb{K}^{p n}$, and so, we have to define another matrix invariant.

The Grassmann manifold $G_{n}\left(\mathbb{K}^{d}\right)$ is the set of $n$-dimensional linear subspaces ( $n$-spaces, for aim of shortness) of $\mathbb{K}^{d}$. The group $\mathbf{U}(d)$ of isometries of $\mathbb{K}^{d}$ acts on $G_{n}\left(\mathbb{K}^{d}\right)^{p}$ on a natural way. We say that two $p$-tuples are isometric if and only if they lie in the same orbit under this action. We say that two $p$-tuples $\left(\Gamma_{1}, \ldots, \Gamma_{p}\right)$ and $\left(\Gamma_{1}^{\prime}, \ldots, \Gamma_{p}^{\prime}\right)$ are congruent if and only if $d\left(\Gamma_{i}, \Gamma_{j}\right)=d\left(\Gamma_{i}^{\prime}, \Gamma_{j}^{\prime}\right)$ (see below for the definition of the geodesic distance $d$ in $G_{n}\left(\mathbb{K}^{d}\right)$ ) for all $i$, $j \in\{1, \ldots, p\}$. Of course, two isometric $p$-tuples are congruent, but the converse is not true.

The problem we investigate here is to find some numerical invariant for $p$ tuples up to isometry. The solution is well known for $p=2$. Given two $n$-spaces $\Gamma_{1}, \Gamma_{2} \subset \mathbb{K}^{d}$, we consider the function $\theta: P\left(\Gamma_{1}\right) \rightarrow \mathbb{R}$ which associates to a line $l$ of $\Gamma_{1}$ the angle between $l$ and its orthogonal projection onto $\Gamma_{2}$. Of course, $\mathbb{K}^{d}$ is endowed with its canonical scalar product $\left(i . e .\left\langle\left(u_{1}, \ldots, u_{d}\right),\left(v_{1}, \ldots, v_{d}\right)\right\rangle=\right.$ 
$\left.\sum_{k=1}^{d} \bar{u}_{k} v_{k}\right)$. So, we can define the angle between two lines $\mathbb{K} u$ and $\mathbb{K} v \in P\left(\mathbb{K}^{d}\right)$ as $\cos ^{-1} \frac{|\langle u, v\rangle|}{\sqrt{\langle u, u\rangle\langle v, v\rangle}}$. The stationary values of the function $\theta$ are called the critical angles between $\Gamma_{1}$ and $\Gamma_{2}$. Critical angles allow us to define the socalled geodesic distance between $\Gamma_{1}$ and $\Gamma_{2}$ as the square root of the sum of the squares of the critical angles.

It can be proven that two pairs of $n$-spaces are isometric if and only if they have the same critical angles. Moreover, given $n$ numbers $\theta_{1}, \ldots, \theta_{n} \in\left[0, \frac{\pi}{2}\right]$, there always exist two $n$-spaces embedded in $\mathbb{K}^{2 n}$ such that these numbers are the critical angles between them.

A convenient way to compute the critical angles between two $n$-spaces $\Gamma_{1}$, $\Gamma_{2} \subset \mathbb{K}^{d}$ is to equip each of them with an orthonormal basis $\left(e_{1}^{i}, \ldots, e_{n}^{i}\right)(i=$ $1,2)$, and to put the coordinates (in the canonical basis of $\mathbb{K}^{d}$ ) of $e_{j}^{i}$ into the $j^{\text {th }}$ column of a $d \times n$ matrix $B^{i}$. Then the cosines of the critical angles are the singular values of the matrix $B_{1}^{*} B_{2}[9]$.

\section{$1.1 \quad$ Earlier results}

If the case of two $n$-spaces is for long quite well-understood, there are hitherto very few investigations concerning triples, or more general $p$-tuples.

Augustin Fruchard investigated the case triples of real planes (i.e. 2-spaces) in [4]. Projecting orthogonally the unit circle of each plane onto the two others yields two ellipses in each plane. The angles between the major axes of these ellipses are called the inner angles. Of course, this notion makes sense if and only if the projection of a unit circle is never a circle, i.e. each pair of planes has two distinct critical angles. A. Fruchard defined a generic triangle as a triple for which the inner angles are well defined, and for which all the angles (critical and inner) belong to $] 0, \frac{\pi}{2}[$. He proved that a generic triangle is fully defined by the six critical angles, the three inner angles, and four signs $\varepsilon_{1}, \varepsilon_{2}, \varepsilon_{3}, \varepsilon_{4}= \pm 1$, the pack of which he refers to as the signature of the triangle. We shall give in section 3 an analogous result, but our method leads to an invariant involving only one sign $\varepsilon$. This is due to the fact that the class of triples we can investigate with our method is slightly larger than the class of generic triples. What appear in A. Fruchard's work as 16 distinct continuous families can be glued into two bigger ones. The triples of the interface are those that can be treated by our method, but are not generic.

Another point of view for the study of a $p$-tuple $\Gamma=\left(\Gamma_{1}, \ldots, \Gamma_{p}\right)$ is to consider the orthogonal projections onto $\Gamma_{1}, \ldots, \Gamma_{p}$. The traces and determinants of various products of these projections are numerical invariants of the isometry class of $\Gamma$. Giovani Masala obtained nice results in this way, still in the case of real planes. He gave a list of invariants which fully characterizes a $p$-tuple. His invariant system allows him to classify the regular quadruples of $G_{2}\left(\mathbb{R}^{8}\right)$. However, although the statements of his theorems differ considerably in spirit from those of A. Fruchard, the proofs are more or less similar. In particular, they chiefly involve the notion of inner angles, and so, let few hope of generalization in higher dimensions [6][7]. 
Another important investigation is the case of $p$-tuples of equi-isoclinic $n$ spaces. Two $n$-spaces are said to be isoclinic if their critical angles are all equal. Further, $p n$-spaces are said to be equi-isoclinic if they are pairwise isoclinic with the same angle. In this quite restrictive case, a theory does exist. A normal Seidel matrix (named after J.J. Seidel in [2]) is a Hermitian $n p \times n p$ matrix with the following property: if we see it as a $p \times p$ matrix whose coefficients are $n \times n$ matrices, then (i) the diagonal coefficients vanish, (ii) the coefficients of the first row and the first column (except the first one which is zero) are identity matrices, and (iii) all other blocks are unitary (or orthogonal if $\mathbb{K}=\mathbb{R}$ ). The unitary (or orthogonal) group acts on the set of normal Seidel matrices by conjugation of each block. The theory provides a way to associate to a $p$-tuple of equi-isoclinic $n$-spaces of angle $\alpha$ one of the orbits of the Seidel matrices under this action. Moreover, the data of this orbit and the angle $\alpha$ fully determine the $p$-tuple. The existence problem has also a nice solution: given a normal Seidel matrix $S$ and an angle $\alpha$, there exist a $p$-tuple of equi-isoclinic $n$-spaces with angle $\alpha$ and associated Seidel matrix $S$ if and only if $\cos \alpha$ is lower than or equal to the opposite of the inverse of the smallest eigenvalue of $S$. In case of equality, the $p$-tuple can be embedded in $\mathbb{K}^{n p-\mu}$, where $\mu$ is the multiplicity of the smallest eigenvalue of $S$; otherwise the $p$-tuple spans $\mathbb{K}^{n p}$ [5][2]. These results were actually stated in the real case, but all the proofs hold verbatim for $\mathbb{K}=\mathbb{C}$.

The method we propose in this article will include as particular cases both A. Fruchard's and J. J. Seidel's points of view.

\subsection{Notation}

Throughout this article, we shall have to deal with $n p \times n p$ matrices which are defined by $n \times n$ blocks. If $M$ is such a matrix, $M_{i j}$ will stand for the $n \times n$ block consisting of the intersection of the lines $(i-1) n+1, \ldots,(i-1) n+n$ and the columns $(j-1) n+1, \ldots,(j-1) n+n$. As usual, if $M$ is a $n \times n$ matrix, $M_{i j}$ is the scalar coefficient which lie on line $i$ and the column $j$. If $M$ and $N$ are $n p \times n p$ matrices, $M * N$ stands for the $n p \times n p$ matrix defined by $(M * N)_{i j}=M_{i j} N_{i j}, 1 \leq i, j \leq p$.

If $X$ is a set of square matrices of order $n$, and $N$ is a diagonal matrix of the same size,

- $\mathbf{M}(k)$ stands for the set of $k \times k$ matrices with coefficients in $\mathbb{K}$.

- $\mathbf{D}(k)$ stands for the set of $k \times k$ diagonal matrices with coefficients in $\mathbb{R}$.

- $\mathbf{D}^{+}(k)$ stands for the set of $k \times k$ diagonal matrices with positive diagonal coefficients.

- $\mathbf{U}(k)$ stands for the unitary group of degree $k$ if $K=\mathbb{C}$, or the orthogonal group if $\mathbb{K}=\mathbb{R}$.

- SU $(k)$ stands for the special unitary group of degree $k$ if $K=\mathbb{C}$, or the special orthogonal group if $\mathbb{K}=\mathbb{R}$. 
- $\mathbf{O}(k)$ stands for the orthogonal group of degree $k$.

- $\mathbf{S O}(k)$ stands for the special orthogonal group of degree $k$.

- $\mathbf{M}(X, p)$ stands for the set of $n p \times n p$ matrices whose diagonal blockcoefficients are $I_{n}$ and other coefficients belongs to $X$.

- $\mathbf{H}(X, p)$ stands for the subset of Hermitian (symmetric if $\mathbb{K}=\mathbb{R}$ ) matrices of $\mathbf{M}(X, p)$.

- $\mathbf{S}(N)$ stands for the set on $n \times n$ matrices whose singular values are the diagonal coefficients of $N$.

- $\mathbf{D}(X, p)$ stands for the set of $n p \times n p$ matrices which are block-diagonal, with diagonal block-coefficients belonging to $X$.

If $M_{1}, \ldots, M_{p}$ belongs to $\mathbf{M}(n)$ we denote by $\operatorname{diag}\left(M_{1}, \ldots, M_{p}\right)$ the blockdiagonal matrix whose diagonal block-coefficients are $M_{1}, \ldots, M_{p}$. As usual, if $\sigma_{1}, \ldots, \sigma_{n}$ are scalars, $\operatorname{diag}\left(\sigma_{1}, \ldots, \sigma_{n}\right) \in \mathbf{D}(n)$ stands for the diagonal matrix with diagonal coefficients $\sigma_{1}, \ldots, \sigma_{n}$.

For $M \in \mathbf{M}(n)$, we denote by $[M]_{p}$ the square matrix of order $n p$ whose block-coefficients are all equal to $M$, save the diagonal ones which are identity matrices.

\section{General theory}

\subsection{A matrix invariant}

Let $\Gamma=\left(\Gamma_{1}, \ldots, \Gamma_{p}\right)$ be a $p$-tuple of $G_{n}\left(\mathbb{K}^{n p}\right)$. The most obvious matrix invariant is a matrix built up from critical angles. We say that a symmetric matrix $\Delta \in \mathbf{M}(\mathbf{D}(n), p)$ is an edge matrix associated to $\Gamma$ if the diagonal coefficients of $\Delta_{i j} \in \mathbf{D}(n)$ are the cosines of the critical angles between $\Gamma_{i}$ and $\Gamma_{j}$. It is clear that a $p$-tuple is, in general, associated to more than one edge-matrix, for the critical angles can be arranged in any order. If we ask furthermore that the cosines are arranged in non-increasing order of multiplicity, and, for cosines of the same multiplicity, in decreasing order, the obtained matrix will be called the - with the definite article - edge matrix of $\Gamma$.

We say that a $p$-tuple is homogeneous if its edge matrix $\Delta$ is such that

i. All of the diagonal coefficients of $\Delta_{i j}$ are positive $(1 \leq i, j \leq p)$.

ii. The set $\mathbf{C} \stackrel{\text { def }}{=}\left\{U \in \mathbf{U}(n) \mid U \Delta_{i j}=\Delta_{j i} U\right\}$ does not depend on $i, j(1 \leq$ $i<j \leq p)$

It is clear that $\mathbf{C}$ is a subgroup of $\mathbf{U}(n)$; we call it the group of $\Delta$. Note that the first point ensures that $\Delta_{i j}$ is invertible.

If the $n$ angles between any two $n$-spaces are pairwise distinct and less than $\frac{\pi}{2}$, the $p$-tuple will be said to be anisoclinic. It is clear that anisoclinic $p$-tuples 
are homogeneous. Indeed, an homogeneous $p$-tuple is anisoclinic if and only if $\mathbf{C}$ is the group of diagonal unitary matrices, if and only if $\mathbf{C}$ is Abelian. Moreover, a generic $p$-tuple of planes is necessarily anisoclinic.

The following Lemma will be useful for further development.

Lemma 1 Let $N \in \mathbf{D}^{+}(n)$. Assume that $U_{1}, V_{1}, U_{2}, V_{2} \in \mathbf{U}(n)$ are such that $U_{1} N V_{1}^{*}=U_{2} N V_{2}^{*}$. Then there exists a matrix $E \in \mathbf{U}(n)$ which commutes with $N$ and such that $U_{2}=U_{1} E$ and $V_{2}=V_{1} E$.

Proof. Let $M=U_{1} N V_{1}^{*}$; we have $M M^{*}=U_{1} N^{2} U_{1}^{*}=U_{2} N^{2} U_{2}^{*}$. Hence the columns of $U_{1}$ and $U_{2}$ are eigenvectors of the same matrix $M M^{*}$. More precisely, if we denote by $B_{i}^{\alpha}$ the block of $U_{i}(i=1,2)$ consisting of eigenvectors associated to one given eigenvalue $\alpha$ of $M M^{*}$, then the columns of $B_{i}^{\alpha}$ form an orthonormal basis of the eigenspace of $M M^{*}$ associated to $\alpha$. Therefore, there exists an unitary matrix $E^{\alpha}$ (whose order is the multiplicity of $\alpha$ ) such that $B_{2}^{\alpha}=B_{1}^{\alpha} E^{\alpha}$. Since the argument holds for any eigenvalue $\alpha$, there exists $E \in \mathbf{U}(n)$ which commute with $N$ and such that $U_{2}=U_{1} E$.

On the other hand, $M^{*} M=V_{1} N^{2} V_{1}^{*}=V_{2} N^{2} V_{2}^{*}$, whence there exists $F \in$ $\mathbf{U}(n)$ such that $V_{2}=V_{1} F$. Now, $U_{1} N V_{1}^{*}=U_{2} N V_{2}^{*}=U_{1} N E F^{*} V_{1}$, whence $E F^{*}=I_{n}$.

From now on, we suppose that $\Gamma$ is homogeneous. Let $\Delta$ be the edge matrix of $\Gamma$, and $\mathbf{C}$ be the group of $\Delta$.

Let each $n$-space $\Gamma_{k}$ be endowed with an orthonormal basis $\left(e_{i}^{k}, \ldots e_{j}^{k}\right)$ and consider the Gram matrix $G$ of the family

$$
\left(e_{1}^{1}, e_{2}^{1}, \ldots, e_{n}^{1}, e_{1}^{2}, \ldots, e_{n-1}^{p}, e_{n}^{p}\right)
$$

As a Gram matrix, $G$ is Hermitian and positive semi-definite. Moreover, due to the fact that $\left(e_{i}^{k}, \ldots e_{j}^{k}\right)$ is orthonormal, $G$ belongs to $\mathbf{H}(\mathbf{M}(n), p)$. If you choose some other orthonormal basis $\left(e_{i}^{\prime k}, \ldots e_{j}^{\prime k}\right)$ in $\Gamma_{k}, 1 \leq k \leq p$, then there exists $V^{k} \in \mathbf{U}(n)$ such that $e_{i}^{\prime k}=\sum_{j=1}^{n} e_{j}^{k} V_{j i}^{k}$. It follows that the corresponding Gram matrix $G^{\prime}$ is obtained from $G$ by the formula $G^{\prime}=D G D^{*}$, where $D \in \mathbf{D}(\mathbf{U}(n), p)$ is defined by $D_{i i}=V^{i *}$. This fact motivates the following definition: two matrices of $\mathbf{H}(\mathbf{M}(n), p)$ are equivalent if they are conjugated by a matrix of $\mathbf{D}(\mathbf{U}(n), p)$. It follows that two Gram matrices are equivalent if and only if they are the matrices of the same $p$-tuple of $n$-spaces, up to a global isometry.

By construction of $G$, the critical angles between $\Gamma_{i}$ and $\Gamma_{j}$ are the singular values of $G_{i j}$, whence there exist $U_{i j}, V_{i j} \in \mathbf{U}(n)$ such that $G_{i j}=U_{i j} \Delta_{i j} V_{i j}^{*}$. Since $G_{i j}=G_{j i}^{*}$, we have $V_{i j} \Delta_{i j} U_{i j}^{*}=U_{j i} \Delta_{j i} V_{j i}^{*}$. By Lemma 1, there exists $C_{j i} \in \mathbf{C}$ such that $U_{i j}=V_{j i} C_{j i}$ and $V_{i j}=U_{j i} C_{j i}$. Moreover, these two formulae infer that $C_{i j} C_{j i}=I_{n}$. It follows that there exist $C \in \mathbf{H}(\mathbf{C}, p)$ and $U \in$ $\mathbf{M}(\mathbf{U}(n), p)$ such that $G=U * C * \Delta * U^{*}$. A Gram matrix which admits such a decomposition will be called a $\Delta$-Gram matrix.

We say that $M \in \mathbf{S}\left(\Delta_{i j}\right)$ is of $L$-kind (respectively $R$-kind) if there exists $W \in \mathbf{U}(n)$ such that $M=W \Delta_{i j}$ (respectively $M=\Delta_{i j} W$ ). It follows from 
Lemma 1 that, if $U, V \in \mathbf{U}(n)$ are such that the matrix $U \Delta_{i j} V^{*}$ is of $L$-kind (respectively $R$-kind), then $V \in \mathbf{C}$ (respectively $U \in \mathbf{C}$ ).

We say that a $\Delta$-Gram matrix $G$ is prereduced if for $j \geq 2, G_{1 j}$ is of $L$-kind, and $G_{12}=\Delta_{12}$. Since $G$ is Hermitian, the prereduceness implies furthermore that $G_{i 1}$ is of $R$-kind for $i \geq 2$, and $G_{21}=\Delta_{21}$.

\section{Lemma 2}

i. Each $\Delta$-Gram matrix is equivalent to a prereduced Gram matrix.

ii. Two prereduced $\Delta$-Gram matrix $G$ and $G^{\prime}$ are equivalent if and only if there exists $D \in \mathbf{D}(\mathbf{C}, p)$ such that $D_{11}=D_{22}$ and $G^{\prime}=D G D^{*}$.

Proof. Chose a decomposition $G=U * C * \Delta * U^{*}$ of $G$. Let $D \in \mathbf{D}(\mathbf{U}(n), p)$ be defined by

$$
\begin{aligned}
D_{11} & =C_{12}^{*} U_{12}^{*} \\
D_{i i} & =U_{i 1}^{*}
\end{aligned} \quad \text { if } i>1,
$$

and put $G^{\prime}=D G D^{*}$. We have for $j>1$,

$$
G_{i j}^{\prime}=D_{i i} G_{i j} D_{j j}^{*}=D_{i i} U_{i j} \Delta_{i j} C_{i j} U_{j i}^{*} U_{j 1} .
$$

Hence $G_{1 i}^{\prime}=D_{11}^{*} U_{1 j} C_{1 j} \Delta_{i j}$ is of $L$-kind and $G_{12}^{\prime}=\Delta_{12}$.

Assume now that $G$ and $G^{\prime}$ are prereduced and equivalent, there exists $D \in$ $\mathbf{D}(\mathbf{U}(n), p)$ such that $G^{\prime}=D G D^{*}$. By hypothesis $G$ is prereduced, so $G_{1 j}$ is of $L$-kind $(j>1)$ and $G_{12}=\Delta_{12}$. It follows that there exist $U_{2}=I_{n}, U_{3}, \ldots, U_{p} \in$ $\mathbf{U}(n)$ such that $G_{1 j}=U_{j} \Delta_{1 j}(j>1)$. Since $G_{1 j}^{\prime}=D_{11} G_{1 j} D_{j j}^{*}=D_{11} U_{j} \Delta_{i j} D_{j j}^{*}$ is of $L$-kind, $D_{j j}^{*} \in \mathbf{C}$ for all $j>1$. Since $G_{12}^{\prime}=D_{11} \Delta_{12} D_{22}^{*}=\Delta_{12}$, by Lemma 1 , we have $D_{11}=D_{22} \in \mathbf{C}$.

It is clear that a decomposition of a $\Delta$-Gram matrix $G$ of the form $U *$ $C * \Delta * U^{*}$ is not unique, and worst, there is no canonical way to define one preferred decomposition among all the possible ones. So it will be necessary to arbitrary distinguish such a decomposition. For this purpose, we choose a (not necessary continuous) function $\mathfrak{s}: \mathbf{U}(n) \rightarrow \mathbf{U}(n)$ such that, for all $U \in \mathbf{U}(n)$, the restriction of $\mathfrak{s}$ to the left coset $U \mathbf{C}$ is constant, and such that $\mathfrak{s}(U) \in$ $U \mathbf{C}$. In other words, we chose in each left coset $U \mathbf{C}$ an arbitrary distinguished element $\mathfrak{s}(U)$. We assume furthermore that $\mathfrak{s}$ is chosen such that $\mathfrak{s}(\mathbf{C})=\left\{I_{n}\right\}$. Such a map $\mathfrak{s}$ will be called a split map. We also define $\delta: \mathbf{U}(n) \rightarrow \mathbf{C}$ by $\delta(U)=\mathfrak{s}(U)^{*} U$, and, for any $U \in \mathbf{U}(n)$, the map $\widehat{U}: \mathbf{C} \rightarrow \mathbf{C}$ defined by $\widehat{U}(E)=\delta(E U)$.

We say that a decomposition $G=U * C * \Delta * U^{*}$ of a $\Delta$-Gram $G$ matrix is $\mathfrak{s - n o r m a l}$ if $U \in \mathbf{M}(\operatorname{Im} \mathfrak{s}, p)$. It is clear that each $\Delta$-Gram matrix has a single $\mathfrak{s}$-normal decomposition. We say that a $\Delta$-Gram matrix is reduced if, on the one hand, it is prereduced, and on the other hand, the first block row of the matrix $C$ of its $\mathfrak{s}$-normal decomposition contains only identity matrix. Note that, since $C$ is Hermitian, the latter condition may be equivalently stated on the first block column. 


\section{Lemma 3}

i. Each $\Delta$-Gram matrix is equivalent to a reduced one.

ii. If two reduced $\Delta$-Gram matrices $G$ and $G^{\prime}$ are equivalent, then there exist $E \in \mathbf{C}$ such that

$$
G^{\prime}=\operatorname{diag}\left(\widehat{U_{11}}(E), \ldots, \widehat{U_{1 p}}(E)\right) G\left(\operatorname{diag}\left(\widehat{U_{11}}(E), \ldots, \widehat{U_{1 p}}(E)\right)\right)^{*},
$$

where $U$ is the first matrix of the $\mathfrak{s}$-normal decomposition of $G$.

Proof. Let $G=U * C * \Delta * U^{*}$ be the $\mathfrak{s - n o r m a l ~ d e c o m p o s i t i o n ~ o f ~ a ~ p r e r e d u c e d ~}$ $\Delta$-Gram matrix $G$. Since $G$ is prereduced, $U_{i 1}=U_{12}=C_{21}=C_{12}=I_{n}$. Define $D \in \mathbf{D}(\mathbf{C}, p)$ by $D_{i i}=C_{1 i}$ for $i \geq 2$. Put $G^{\prime}=D G D^{*}$; define $U^{\prime}$ and $C^{\prime}$ such that $G^{\prime}=U^{\prime} * C^{\prime} * \Delta * U^{\prime *}$ is the s-normal decomposition of $G^{\prime}$. Since $D_{11}=I_{n}=D_{12}$, by virtue of Lemma 2 , the matrix $G^{\prime}$ is prereduced. Moreover, for $i \geq 2, G_{i 1}^{\prime}=D_{i i} U_{i 1} C_{i 1} \Delta_{1 i} U_{1 i}^{*} D_{11}^{*}=C_{1 i} C_{i 1} \Delta_{1 i} U_{1 i}^{*}$, whence $C_{i 1}^{\prime}=I_{n}$.

Now assume that $G=U * C * \Delta * U^{*}$ and $G^{\prime}=D G D^{*}=U^{\prime} * C^{\prime} * \Delta * U^{\prime *}$ are reduced and that the above decompositions are $\mathfrak{s}$-normal. By virtue of Lemma 2 , $D \in \mathbf{D}(\mathbf{C}, p)$. Moreover $G_{i 1}^{\prime}=D_{i i} \Delta_{i 1}\left(D_{11} U_{1 i}\right)^{*}$ and $I_{n}=C_{1 i}^{\prime}=D_{i i} \widehat{U_{1 i}}\left(D_{11}\right)^{*}$, thus $D_{i i}=\widehat{U_{1 i}}\left(D_{11}\right)$.

We define a $\mathfrak{s}$-pair as an ordered pair $(U, C) \in \mathbf{M}(\operatorname{Im} \mathfrak{s}, p) \times \mathbf{H}(\mathbf{C}, p)$ such that for all $i \in\{1, \ldots, p\}, U_{i 1}=C_{i 1}=C_{1 i}=U_{12}=I_{n}$. If $G=U * C * \Delta_{p} * U^{*}$ is a $\mathfrak{s}$-normal decomposition of a reduced $\Delta$-Gram matrix $G$ associated to $\Gamma$, then $(U, C)$ is a $\mathfrak{s}$-pair, which is said to be associated to $\Gamma$. We define an action of $\mathbf{C}$ on the set of $\mathfrak{s}$-pairs by $E \cdot(U, C)=(E \cdot U, E \cdot C)$, where

$$
\begin{aligned}
& (E \cdot U)_{i j}=\mathfrak{s}\left(\widehat{U_{1 i}}(E) U_{i j}\right) \\
& (E \cdot C)_{i j}=\widehat{U_{i j}} \widehat{U_{1 i}}(E) C_{i j}\left(\widehat{U_{j i}} \circ \widehat{U_{1 j}}(E)\right)^{*} .
\end{aligned}
$$

Note that the notation $E \cdot C$ is abusive, for $E \cdot C$ actually depends on $U$. A thorough verification shows that $E \cdot(U, C)$ is the $\mathfrak{s}$-pair corresponding to the Gram matrix $D\left(U * C * \Delta * U^{*}\right) D^{*}$, where $D$ is the block-diagonal matrix defined by $D_{i i}=\widehat{U_{1 i}}(E)$. Hence, by virtue of Lemma 3 , two $\mathfrak{s}$-pairs are associated to the same $p$-tuples if and only if they lie in the same orbit. An orbit under this action will be called a $\mathfrak{s - o r b i t . ~ T h e ~ f o l l o w i n g ~ T h e o r e m ~ s u m m a r i z e ~ t h e ~ a b o v e ~}$ considerations.

Theorem 1 Two homogeneous p-tuple of $G_{n}\left(\mathbb{K}^{n p}\right)$ are isometric if and only if they have the same edge matrix, and the same $\mathfrak{s}$-orbit.

Remark 4 In the case of an p-tuple $\Gamma$ of equi-isoclinic n-spaces of angle $\alpha$, $\Delta_{i j}=\cos (\alpha) I_{n}, \mathbf{C}=\mathbf{U}(n)$ and $\mathfrak{s}=1$. If $(U, C)$ is a $\mathfrak{s}$-pair associated to $\Gamma$, then $U=\left[I_{n}\right]_{p}$. It follows that the p-tuple is wholly determined by the orbit of the matrix $C \in \mathbf{H}(\mathbf{U}(n), p)$. The matrix $C-I_{n p}$ is the normal Seidel matrix mentioned in the introduction. See section 5.2. 
Remark $\mathbf{5}$ The center $\mathbf{Z}$ of $\mathbf{U}(p)$ (which is clearly a normal subgroup of $\mathbf{C}$ ) fixes any $\mathfrak{s}$-pair. Hence this action can be seen as an action of $\mathbf{C} / \mathbf{Z}$.

In the special case where $U=\left[I_{n}\right]_{p}$, we say that the $\mathfrak{s}$-pair $(U, C)$ is special. On the other hand, if $U$ contains exactly $2 p$ (i.e. the minimum number) blockcoefficients equal to $I_{n}$, then $(U, C)$ is said to be common. Of course, a $\mathfrak{s}$-pair may be neither common nor special. Note that, if one $\mathfrak{s}$-pair is common, then all the $\mathfrak{s}$-pairs of its $\mathfrak{s}$-orbit are also common; in this case the corresponding $\mathfrak{s - o r b i t ~ a n d ~} p$-tuples are said to be common.

Proposition 6 Assume that $\mathbf{C}$ is Abelian. If the $\mathfrak{s}$-pair $(U, C)$ is special, then its $\mathfrak{s}$-orbit is a singleton.

Proof. Let $\left(U=\left[I_{n}\right]_{p}, C\right)$ be a special s-pair and choose $E \in \mathbf{C}$, we have $(E \cdot U)_{i j}=\mathfrak{s}\left(\widehat{I_{n}}(E) I_{n}\right)=\mathfrak{s}(E)=I_{n}$, whence $E \cdot U=U$. Moreover $(E \cdot C)_{i j}=$ $\operatorname{id}_{\mathbf{C}} \circ \operatorname{id}_{\mathbf{C}}(E) C_{i j}\left(\operatorname{id}_{\mathbf{C}} \circ \operatorname{id}_{\mathbf{C}}(E)\right)^{*}=E C_{i j} E^{*}=C_{i j}$. Hence $E \cdot(U, C)=(U, C)$.

\subsection{Regularity}

A $p$-tuple $\Gamma=\left(\Gamma_{1}, \ldots, \Gamma_{p}\right)$ is said to be regular, if it is isometric to $\sigma \Gamma \stackrel{\text { def }}{=}$ $\left(\Gamma_{\sigma(1)}, \ldots, \Gamma_{\sigma(p)}\right)$ for any permutation $\sigma \in S_{p}$. The aim of this section is to give some characterization of regular $p$-tuples by mean of its $\mathfrak{s}$-pair. Of course, the critical angles between any two $n$-spaces of a regular $p$-tuple do not depend on the considered pair of $n$-spaces. In other words, the $p$-tuple admits an edge matrix in which all the non-diagonal block are identical.

Theorem 2 Let $\Delta=[N]_{p}$ be an edge matrix in which all the non-diagonal blocks are equal to $N \in \mathbf{D}^{+}(n)$. A p-tuple with edge matrix $\Delta$ and $\mathfrak{s}$-pair $(U, C)$ is regular if and only if there exist $F_{1}, F_{3}, \ldots, F_{n} \in \mathbf{C}$ such that

$$
\begin{aligned}
& U_{2 j}=\mathfrak{s}\left(F_{1} U_{1 j}\right) \quad(3 \leq j \leq p) \\
& U_{1 j}=\mathfrak{s}\left(F_{1} U_{2 j}\right) \quad(3 \leq j \leq p) \\
& \mathfrak{s}\left(C_{2 j} U_{j 2}^{*}\right)=\mathfrak{s}\left(\widehat{U_{1 j}}\left(F_{1}\right) U_{j 2}\right) \quad(3 \leq j \leq p) \\
& \mathfrak{s}\left(C_{2 i} U_{i 2}^{*} U_{i j}\right)=\mathfrak{s}\left(\widehat{U_{1 i}}\left(F_{1}\right) U_{i j}\right) \quad(3 \leq i \neq j \leq p) \\
& \widehat{U_{j 2}^{*}}\left(C_{2 j}\right)^{*}=\widehat{U_{2 j}}\left(F_{1}\right) C_{2 j}\left(\widehat{U_{j 2}} \circ \widehat{U_{1 j}}\left(F_{1}\right)\right)^{*} \\
& (3 \leq j \leq p) \\
& \widehat{U_{i 2}^{*} U_{i j}}\left(C_{2 i}\right) C_{i j}\left(\widehat{U_{j 2}^{*} U_{j i}}\left(C_{2 j}\right)\right)^{*}=\widehat{U_{i j}} \circ \widehat{U_{1 i}}\left(F_{1}\right) C_{i j}\left(\widehat{U_{j i}} \circ \widehat{U_{1 j}}\left(F_{1}\right)\right)^{*} \\
& (3 \leq i \neq j \leq p) \\
& \mathfrak{s}\left(F_{k} U_{1 k}\right)=\mathfrak{s}\left(U_{1 k}^{*}\right) \\
& (3 \leq k \leq p) \\
& \mathfrak{s}\left(F_{k} U_{1 j}\right)=\mathfrak{s}\left(U_{1 k}^{*} U_{1 j}\right) \quad(3 \leq k \neq j \leq p)
\end{aligned}
$$




$$
\begin{aligned}
& \mathfrak{s}\left(F_{k} U_{2 k}\right)=U_{k 2} \quad(3 \leq k \leq p) \\
& \mathfrak{s}\left(F_{k} U_{2 j}\right)=U_{k j} \quad(3 \leq k \neq j \leq p) \\
& \mathfrak{s}\left(\widehat{U_{1 k}}\left(F_{k}\right) U_{k 2}\right)=\mathfrak{s}\left(\delta\left(U_{1 k}^{*}\right) U_{2 k}\right) \quad(3 \leq k \leq p) \\
& \mathfrak{s}\left(\widehat{U_{1 i}}\left(F_{k}\right) U_{i 2}\right)=\mathfrak{s}\left(\delta\left(U_{1 k}^{*} U_{1 i}\right) U_{i k}\right) \quad(3 \leq k \neq i \leq p) \\
& \mathfrak{s}\left(\widehat{U_{1 k}}\left(F_{k}\right) U_{k j}\right)=\mathfrak{s}\left(\delta\left(U_{1 k}^{*}\right) U_{2 j}\right) \quad(3 \leq k \neq j \leq p) \\
& \mathfrak{s}\left(\widehat{U_{1 i}}\left(F_{k}\right) U_{i k}\right)=\mathfrak{s}\left(\delta\left(U_{1 k}^{*} U_{1 i}\right) U_{i 2}\right) \quad(3 \leq k \neq i \leq p) \\
& \mathfrak{s}\left(\widehat{U_{1 i}}\left(F_{k}\right) U_{i j}\right)=\mathfrak{s}\left(\delta\left(U_{1 k}^{*} U_{1 i}\right) U_{i j}\right) \\
& (3 \leq i, j, k \leq p, i \neq j, j \neq k, k \neq i) \\
& \widehat{U_{2 k}}\left(F_{k}\right) C_{2 k}\left(\widehat{U_{k 2}} \circ \widehat{U_{1 k}}\left(F_{k}\right)\right)^{*}=C_{k 2} \widehat{U_{2 k}}\left(\delta\left(U_{1 k}^{*}\right)\right)^{*} \quad(3 \leq k \leq p) \\
& \widehat{U_{2 j}}\left(F_{k}\right) C_{2 j}\left(\widehat{U_{j 2}} \circ \widehat{U_{1 j}}\left(F_{k}\right)\right)^{*}=C_{k j} \widehat{U_{j k}}\left(\delta\left(U_{1 k}^{*} U_{1 j}\right)\right)^{*} \\
& (3 \leq k \neq j \leq p) \\
& \widehat{U_{k j}} \circ \widehat{U_{1 k}}\left(F_{k}\right) C_{k j}\left(\widehat{U_{j k}} \circ \widehat{U_{1 j}}\left(F_{k}\right)\right)^{*}=\widehat{U_{2 j}}\left(\delta\left(U_{1 k}^{*}\right)\right) C_{2 j} \widehat{U_{j 2}}\left(\delta\left(U_{1 k}^{*} U_{1 j}\right)\right)^{*} \\
& (3 \leq k \neq j \leq p) \\
& \widehat{U_{i j}} \circ \widehat{U_{1 i}}\left(F_{k}\right) C_{i j}\left(\widehat{U_{j i}} \circ \widehat{U_{1 j}}\left(F_{k}\right)\right)^{*}=\widehat{U_{i j}}\left(\delta\left(U_{1 k}^{*} U_{1 i}\right)\right) C_{i j} \widehat{U_{j i}}\left(\delta\left(U_{1 k}^{*} U_{1 j}\right)\right)^{*} \\
& (3 \leq i, j, k \leq p, i \neq j, j \neq k, k \neq i) .
\end{aligned}
$$

Proof. Let $\left(\Gamma_{1}, \ldots, \Gamma_{p}\right)$ be a $p$-tuple of edge matrix $\Delta=[N]_{p}$ and $\mathfrak{s}$-pair $(U, C)$, where $\mathfrak{s}$ is a split map with respect to the group $\mathbf{C}$ of $\Delta$. For $k \neq 2$, we denote by $G^{k}$ be the matrix obtained from $G \stackrel{\text { def }}{=} U * C * \Delta * U^{*}$ by exchanging the block lines of indices 2 and $k$, and the corresponding block columns. Clearly $G^{k}$ is a Gram matrix corresponding to the $p$-tuple $(2 k) \Gamma$. Since $S_{p}$ is generated by the the transpositions $(12),(23), \ldots,(2 p), \Gamma$ is regular if and only if the s-pairs $\left(U^{k}, C^{k}\right)$ corresponding to $G^{k}(k=1,3,4, \ldots, p)$ lie in the same orbit as $(U, C)$. From now on, $i$ and $j$ are indices distinct from 1 and 2 , and distinct to each other. By definition of $G^{1}$ we have

$$
G_{12}^{1}=N, G_{1 j}^{1}=U_{2 j} C_{2 j} N U_{j 2}^{*}, G_{2 j}^{1}=U_{1 j} N, G_{i j}^{1}=U_{i j} C_{i j} N U_{j i}^{*} .
$$

Define

$$
G^{1^{\prime}} \stackrel{\text { def }}{=} \operatorname{diag}\left(U_{12}^{*}, \ldots, U_{n 2}^{*}\right) G^{1} \operatorname{diag}\left(U_{12}^{*}, \ldots, U_{n 2}^{*}\right)^{*}
$$

we obtain

$$
\begin{aligned}
& G_{12}^{1^{\prime}}=N, G_{1 j}^{1^{\prime}}=U_{12}^{*} U_{2 j} C_{2 j} N U_{j 2}^{*} U_{j 2}=U_{2 j} C_{2 j} N, \\
& G_{2 j}^{1^{\prime}}=U_{1 j} N U_{j 2}, G_{i j}^{1^{\prime}}=U_{i 2}^{*} U_{i j} C_{i j} N U_{j i}^{*} U_{j 2} .
\end{aligned}
$$

Put

$$
G^{1^{\prime \prime}} \stackrel{\text { def }}{=} \operatorname{diag}\left(C_{21}, \ldots, C_{2 n}\right) G^{1^{\prime}} \operatorname{diag}\left(C_{21}, \ldots, C_{2 n}\right)^{*}
$$


we have

$$
\begin{aligned}
& G_{12}^{1^{\prime \prime}}=N, G_{1 j}^{1^{\prime \prime}}=U_{2 j} N, G_{2 j}^{1^{\prime \prime}}=U_{1 j} N\left(C_{2 j} U_{j 2}^{*}\right)^{*}, \\
& G_{i j}^{1^{\prime \prime}}=C_{2 i} U_{i 2}^{*} U_{i j} C_{i j} N\left(C_{2 j} U_{j 2}^{*} U_{j i}\right)^{*} .
\end{aligned}
$$

The matrix $G^{1^{\prime \prime}}$ is reduced, it follows that

$$
\begin{aligned}
U_{1 j}^{1} & =U_{2 j}, U_{2 j}^{1}=U_{1 j}, U_{i 2}^{1}=\mathfrak{s}\left(C_{2 j} U_{j 2}^{*}\right) \\
U_{i j}^{1} & =\mathfrak{s}\left(C_{2 i} U_{i 2}^{*} U_{i j}\right), C_{2, j}^{1}=\widehat{U_{j 2}^{*}}\left(C_{2 j}\right)^{*} \\
C_{i, j}^{1} & =\widehat{U_{i 2}^{*} U_{i j}}\left(C_{2 i}\right) C_{i j}\left(\widehat{U_{j 2}^{*} U_{j i}}\left(C_{2 j}\right)\right)^{*} .
\end{aligned}
$$

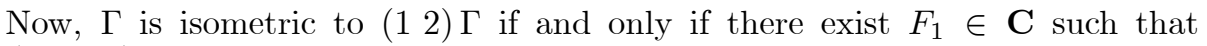
$\left(U^{1}, C^{1}\right)=F_{1} \cdot(U, C)$, i.e. such that the relations (3), ., (8) hold.

Let $k$ be fixed in $\{3, \ldots, p\}$. From now on, $i$ and $j$ are moreover supposed to be distinct from $k$. By definition of $G^{k}$, we have

$$
\begin{aligned}
& G_{12}^{k}=U_{1 k} N, G_{1 j}^{k}=U_{1 j} N, G_{1 k}^{k}=N, G_{2 j}^{k}=U_{k j} C_{k j} N U_{j k}^{*}, \\
& G_{2 k}^{k}=U_{k 2} C_{k 2} N U_{2 k}^{*}, G_{k j}^{k}=U_{2 j} C_{2 j} N U_{j 2}^{*}, G_{i j}^{k}=U_{i j} C_{i j} N U_{j i}^{*} .
\end{aligned}
$$

Put

$$
G^{k^{\prime}} \stackrel{\text { def }}{=} \operatorname{diag}\left(U_{1 k}^{*}, I_{n}, \ldots, I_{n}\right) G^{k} \operatorname{diag}\left(U_{1 k}^{*}, I_{n}, \ldots, I_{n}\right)^{*},
$$

we obtain

$$
\begin{aligned}
& G_{12}^{k^{\prime}}=N, G_{1 j}^{k^{\prime}}=U_{1 k}^{*} U_{1 j} N, G_{1 k}^{k^{\prime}}=U_{1 k}^{*} N, G_{2 j}^{k^{\prime}}=U_{k j} C_{k j} N U_{j k}^{*}, \\
& G_{2 k}^{k^{\prime}}=U_{k 2} C_{k 2} N U_{2 k}^{*}, G_{k j}^{k^{\prime}}=U_{2 j} C_{2 j} N U_{j 2}^{*}, G_{i j}^{k^{\prime}}=U_{i j} C_{i j} N U_{j i}^{*} .
\end{aligned}
$$

Let $D$ be the block-diagonal matrix defined by $D_{11}=D_{22}=I_{n}, D_{k k}=\delta\left(U_{1 k}^{*}\right)$ and $D_{j j}=\delta\left(U_{1 k}^{*} U_{1 j}\right)$. Put $G^{k^{\prime \prime}} \stackrel{\text { def }}{=} D G^{k} D^{*}$, we have

$$
\begin{aligned}
& G_{12}^{k^{\prime \prime}}=N, G_{1 j}^{k^{\prime \prime}}=\mathfrak{s}\left(U_{1 k}^{*} U_{1 j}\right) N, G_{1 k}^{k^{\prime \prime}}=\mathfrak{s}\left(U_{1 k}^{*}\right) N, \\
& G_{2 j}^{k^{\prime \prime}}=U_{k j} C_{k j} N\left(\delta\left(U_{1 k}^{*} U_{1 j}\right) U_{j k}\right)^{*}, G_{2 k}^{k^{\prime \prime}}=U_{k 2} C_{k 2} N\left(\delta\left(U_{1 k}^{*}\right) U_{2 k}\right)^{*}, \\
& G_{k j}^{k^{\prime \prime}}=\delta\left(U_{1 k}^{*}\right) U_{2 j} C_{2 j} N\left(\delta\left(U_{1 k}^{*} U_{1 j}\right) U_{j 2}\right)^{*}, \\
& G_{i j}^{k^{\prime \prime}}=\delta\left(U_{1 k}^{*} U_{1 i}\right) U_{i j} C_{i j} N\left(\delta\left(U_{1 k}^{*} U_{1 j}\right) U_{j i}\right)^{*} .
\end{aligned}
$$

The matrix $G^{k^{\prime \prime}}$ is reduced, whence

$$
\begin{aligned}
U_{1 k}^{k} & =\mathfrak{s}\left(U_{1 k}^{*}\right), U_{1 j}^{k}=\mathfrak{s}\left(U_{1 k}^{*} U_{1 j}\right), U_{2 k}^{k}=U_{k 2}, U_{2 j}^{k}=U_{k j}, \\
U_{k 2}^{k} & =\mathfrak{s}\left(\delta\left(U_{1 k}^{*}\right) U_{2 k}\right), U_{i 2}^{k}=\mathfrak{s}\left(\delta\left(U_{1 k}^{*} U_{1 i}\right) U_{i k}\right), U_{k j}^{k}=\mathfrak{s}\left(\delta\left(U_{1 k}^{*}\right) U_{2 j}\right), \\
U_{i k}^{k} & =\mathfrak{s}\left(\delta\left(U_{1 k}^{*} U_{1 i}\right) U_{i 2}\right), U_{i j}^{k}=\mathfrak{s}\left(\delta\left(U_{1 k}^{*} U_{1 i}\right) U_{i j}\right), \\
C_{2 k}^{k} & =C_{k 2} \widehat{U_{2 k}}\left(\delta\left(U_{1 k}^{*}\right)\right)^{*}, C_{2 j}^{k}=C_{k j} \widehat{U_{j k}}\left(\delta\left(U_{1 k}^{*} U_{1 j}\right)\right)^{*}, \\
C_{k j}^{k} & =\widehat{U_{2 j}}\left(\delta\left(U_{1 k}^{*}\right)\right) C_{2 j} \widehat{U_{j 2}}\left(\delta\left(U_{1 k}^{*} U_{1 j}\right)\right)^{*}, \\
C_{i j}^{k} & =\widehat{U_{i j}}\left(\delta\left(U_{1 k}^{*} U_{1 i}\right)\right) C_{i j} \widehat{U_{j i}}\left(\delta\left(U_{1 k}^{*} U_{1 j}\right)\right)^{*} .
\end{aligned}
$$


Now, $\Gamma$ is isometric to $(2 k) \Gamma$ if and only if there exist $F_{k} \in \mathbf{C}$ such that $\left(U^{k}, C^{k}\right)=E \cdot(U, C)$, i.e. such that the relations $(9), \ldots,(21)$ hold.

We say that a $\mathfrak{s}$-pair is regular, if it is associated to some regular $p$-tuple. By Theorems 2 and 4 (see below), it is equivalent to say that it satisfies the relations $(3), \ldots,(21)$. A $\mathfrak{s}$-orbit will be said to be regular if one (or equivalently each) of its $\mathfrak{s}$-pair is regular.

Proposition 7 A regular p-tuple is either common of special.

Proof. Follows from relations (3), (11), (12) and (15).

Theorem 3 Let $\Gamma$ be a special anisoclinic p-tuple with edge matrix $\Delta=[N]_{p}$ and $\mathfrak{s}$-pair $\left(\left[I_{n}\right]_{p}, C\right)$. Then $\Gamma$ is regular if and only if there exist $E \in \mathbf{C}$ such that $E^{2}=I_{n}$ and $C_{i j}=E$ for $2 \leq i \neq j \leq p$

Proof. Put $U_{i j}=I_{n}$ in the relations (3), ., (21). The relations (3), ., (6), $(9), \ldots,(17)$ and $(21)$ are always satisfied. The others become

$$
\begin{aligned}
C_{2 j}^{*} & =C_{2 j}, & & (3 \leq j \leq p) \\
C_{2 i} C_{i j} C_{2 j} & =C_{i j}, & & (3 \leq i \neq j \leq p) \\
C_{2 k} & =C_{k 2}, & & (3 \leq k \leq p) \\
C_{2 j} & =C_{k j}, & & (3 \leq k \neq j \leq p) \\
C_{k j} & =C_{2 j}, & & (3 \leq k \neq j \leq p)
\end{aligned}
$$

From (25), $C_{k j}$ does not depends on $k=2, \ldots, p, k \neq j$. From (22), $C_{k j}$ is Hermitian. On the other hand, $C$ is also Hermitian, whence $C_{k j}$ does not depends on $j$ either. Conversely, if all the $C_{i j}$ are equal for $2 \leq i \neq j \leq p$, the relations $(22), \ldots,(26)$ are satisfied.

\subsection{The existence problem}

The problem of existence of a $p$-tuple with prescribed edge matrix $\Delta$ and $\mathfrak{s}$-pair $(U, C)$ admits an obvious solution: such a $p$-tuple will exist if and only if the matrix $U * C * \Delta * U^{*}$ is positive semi-definite. The criterion can be restated in signs of some minors of the matrix, and so appears to be as easy as the Euclidean case. It seems difficult to expect a better one in such a general theory. However, the criterion is no so easy to use in practice, so it will be convenient to have some sufficient conditions. We shall prove in particular that a $p$-tuple with a small enough edge matrix (i.e. large enough prescribed critical angles) always exists. We says that a matrix $M$ is lower than or equal to a matrix $N$, and write $M \leq N$, if and only if each scalar coefficient of $M$ is lower than or equal to its corresponding coefficient in $N$.

Theorem 4 For any integers $n \geq 1, p \geq 2$ there exits a positive number $\sigma$, which depends only on $n$ and $p$, such that for any $\mathfrak{s}$-pair $(U, C)$ and any edgematrix $\Delta \in \mathbf{M}\left(\mathbf{D}^{+}(n), p\right)$ lower than or equal to $[\operatorname{diag}(\sigma, \ldots, \sigma)]_{p}$, there exist a p-tuple which is associates to them. 
Proof. If $H$ is an Hermitian matrix, we denote by $l(H)$ its smallest eigenvalue. It is well known that the map $l$ hereby defined is continuous. For any $\mathfrak{s}$-pair $(U, C)$ and edge matrix $\Delta$, we have

$$
l\left(U * C * \Delta * U^{*}\right) \geq \min _{\substack{V \in \mathbb{M}(\mathbf{U}(n), p) \\ K \in \mathbf{H}(\mathbf{U}(n), p)}} l\left(V * K * \Delta * V^{*}\right) \stackrel{\text { def }}{=} L(\Delta) .
$$

Since $\mathbf{U}(n)$ is compact, it is clear that the minimum exists and that the function $L$ is continuous. Moreover, $L\left([0]_{p}\right)=1$, whence there exists $\sigma$ such that, for any edge matrix $\Delta \leq[\operatorname{diag}(\sigma, \ldots, \sigma)]_{p}, L(\Delta) \geq 0$. Hence, for any s-pair $(U, C)$, and any $\Delta$ satisfying the above inequality, we have $l\left(U * C * \Delta * U^{*}\right) \geq 0$, i.e. $U * C * \Delta * U^{*}$ is a Gram matrix.

Remark 8 Augustin Fruchard proved in [4] that, for $\mathbb{K}=\mathbb{R}, p=3$ and $n=2$, $\frac{1}{3}$ is the best possible value for $\sigma$.

The second Theorem of this section states that, roughly speaking, if we choose a $\mathfrak{s}$-pair and the critical angles of one edge of the $p$-tuple, then it will exist, provided that the other critical angles are large enough. We need for its proof the (rather well-known) following Lemma. As for its proof, we refer, for instance, to [8].

Lemma 9 Let $A, B, C, D$ be four square matrices of order $k$. Let $M$ be the matrix of order $2 k$ defined by

$$
M=\left(\begin{array}{cc}
A & B \\
C & D
\end{array}\right)
$$

If $C D=D C$, then $\operatorname{det} M=\operatorname{det}(A D-B C)$.

Theorem 5 Let $N \in \mathbf{D}^{+}(n)$ be such that $\left.N_{i i} \in\right] 0,1[(1 \leq i \leq n)$. Choose distinct indices $i_{0}$ and $j_{0}$ in $\{1, \ldots, p\}$. Let $\mathcal{E}(\gamma)$ be the set of $n p \times n p$ edge matrix $\Delta$ such that $\Delta_{i_{0} j_{0}}=\Delta_{j_{0} i_{0}}=N$ and $\Delta_{i j} \leq \operatorname{diag}(\gamma, \ldots, \gamma)$ for $i \neq j$ and $\{i, j\} \neq\left\{i_{0}, j_{0}\right\}$. Let $\mathbf{C}$ be the group of unitary matrices commuting with $N$ and $\mathfrak{s}$ a split map with respect to $\mathbf{C}$. For any $\mathfrak{s}$-pair $(U, C)$ there exists $\gamma>0$ such that, for any $\Delta \in \mathcal{E}(\gamma)$, a p-tuple with Gram matrix $U * C * \Delta * U^{*}$ exists.

Proof. Let $\Delta_{0} \in \mathbf{M}(\mathbf{D}(n), p)$ be such that $\left(\Delta_{0}\right)_{i_{0} j_{0}}=\left(\Delta_{0}\right)_{j_{0} i_{0}}=N$ and $\left(\Delta_{0}\right)_{i j}=0 \in \mathbf{M}(n)$ for $1 \leq i \neq j \leq p,\{i, j\} \neq\left\{i_{0}, j_{0}\right\}$. Since the eigenvalues of a matrix depends continuously of its coefficients, it is sufficient to prove that $G=U * C * \Delta_{0} * U^{*}$ is positive definite. Put $A \stackrel{\text { def }}{=} G_{i_{0} j_{0}}=U_{i_{0} j_{0}} C_{i_{0} j_{0}} N U_{j_{0} i_{0}}^{*}$, we have $A A^{*}=U_{i_{0} j_{0}}^{2} \Delta_{i_{0} j_{0}}^{2} U_{i_{0} j_{0}}^{*}$. Rearranging lines and columns, and by virtue 
of Lemma 9, we have

$$
\begin{aligned}
\operatorname{det}\left(G-x I_{n}\right) & =\left|\begin{array}{ccccc}
(1-x) I_{n} & A & 0 & \cdots & 0 \\
A^{*} & (1-x) I_{n} & 0 & \cdots & 0 \\
0 & 0 & \ddots & \ddots & \vdots \\
\vdots & \vdots & \ddots & (1-x) I_{n} & 0 \\
0 & 0 & \cdots & 0 & (1-x) I_{n}
\end{array}\right| \\
& =(1-x)^{(p-2) n}\left|\begin{array}{cc}
(1-x) I_{n} & A \\
A^{*} & (1-x) I_{n}
\end{array}\right| \\
& =(1-x)^{(p-2) n} \operatorname{det}\left((1-x)^{2} I_{n}-U_{i_{0} j_{0}} \Delta_{i_{0} j_{0}}^{2} U_{i_{0} j_{0}}^{*}\right) \\
& =(1-x)^{(p-2) n} \operatorname{det}\left((1-x)^{2} I_{n}-\Delta_{i_{0} j_{0}}^{2}\right) \\
& =(1-x)^{(p-2) n} \prod_{i=1}^{n}\left(1-\sigma_{i}-x\right)\left(1+\sigma_{i}-x\right) .
\end{aligned}
$$

It follows that the eigenvalues of $G$ are $1,1-\sigma_{i}$ and $1+\sigma_{i}$, and so are all positive.

The last theorem of this section concerns special regular $p$-tuple.

Theorem 6 Let $\sigma_{1}, \ldots, \sigma_{n}$ be pairwise distinct numbers of $] 0,1[$. Let $\mathbf{C}$ be the group of diagonal unitary matrices of order $n$. Let $E$ be an element of $\mathbf{C}$ such that $E^{2}=I_{n}$ (i.e. $E \in \mathbf{D}(n)$ and $E_{i i}= \pm 1$ ). Let $C \in H(\mathbf{C}, p)$ be defined by $C_{1 j}=I_{n}(1 \leq j \leq p)$ and $C_{i j}=E(2 \leq i \neq j \leq p)$. Then the (special regular anisoclinic) p-tuple with edge matrix $\left[\operatorname{diag}\left(\sigma_{1}, \ldots, \sigma_{n}\right)\right]_{p}$ and $\mathfrak{s}$-pair $\left(\left[I_{n}\right]_{p}, C\right)$ exists if and only if, for each index $i$, either $E_{i i}=1$ or $\sigma_{i} \leq \frac{1}{p-1}$.

Proof. Put $N \stackrel{\text { def }}{=} \operatorname{diag}\left(\sigma_{1}, \ldots, \sigma_{n}\right)$ and $G=C *[N]_{p}$. The $p$-tuple with edge matrix $[N]_{p}$ and $\mathfrak{s}$-pair $\left(\left[I_{n}\right]_{p}, C\right)$ exists if and only if $G$ is positive semi-definite. Note that $G$ and $[N E]_{p}$ are conjugated by $\operatorname{diag}\left(E, I_{n}, \ldots, I_{n}\right)$ and thus have the same spectrum. Denote by $J_{p}$ the $p \times p$ matrix whose diagonal coefficients are 0 and the others are 1 . we have $[N E]_{p}=I_{n p}+J_{p} \otimes N E$, and so the eigenvalues of $G$ are the product of eigenvalues of $J_{p}$ by eigenvalues of $N E$, plus 1 . The computation of the eigenvalues of $J_{p}$ is classical, they are -1 (with multiplicity $p-1)$ and $p-1$. Hence the eigenvalues of $G$ are $1-\sigma_{1} E_{11}, \ldots, 1-\sigma_{p} E_{p p}$ (multiplicity $p-1)$ and $1+(p-1) \sigma_{1} E_{11}, \ldots, 1+(p-1) \sigma_{p} E_{p p}$. The $p$ first eigenvalues are non-negative, the non-negativeness of the other ones yields the conditions $E_{i i}=1$ or $\sigma_{i} \leq \frac{1}{p-1}$.

\section{The real anisoclinic case}

In this section we consider only real anisoclinic $p$-tuples. In this case, the group $\mathbf{C}$ is the group of diagonal matrices whose diagonal coefficients are \pm 1 , or in 
other words, the group generated by the reflections with respect to the hyperplanes orthogonal to the vectors of the canonical basis. Obviously $\mathbf{C}$ is of order $2^{n}$, and the center $\mathbf{Z}$ of $\mathbf{O}(n)$ is $\left\{ \pm I_{n}\right\}$, hence the $\mathfrak{s}$-orbits have at most $2^{n-1}$ s-pairs.

\subsection{Case of planes}

In this section, we only consider $p$-tuples of planes, i.e. $n=2$. We denote by $R(\alpha)$ the rotation of angle $\alpha$ and by $S$ the symmetry with respect to the axis $\mathbb{R}(1,0)$. It is well known that $\mathbf{O}(2)=\{R(\alpha), R(\alpha) S \mid \alpha \in \mathbb{R} / 2 \pi \mathbb{Z}\}$. The group $\mathbf{C}$ has four elements, namely $\pm I_{2}, \pm S$, and the left coset of $R(\alpha)$ is $\{R(\alpha), R(\alpha+\pi), R(\alpha) S, R(\alpha+\pi) S\}$. We define $\mathfrak{s}$ by

$$
\begin{array}{llrl}
\mathfrak{s}(R(\alpha) S) & =\mathfrak{s}(R(\alpha))=R(\alpha), & & \text { if } \alpha \in[0, \pi[, \\
\mathfrak{s}(R(\alpha) S)=\mathfrak{s}(R(\alpha))=R(\alpha+\pi), & & \text { otherwise. }
\end{array}
$$

Of course, the interval $[0, \pi$ [ should be understood as an interval of the circle $\mathbb{R} / 2 \pi \mathbb{Z}$. It's clear that this function $\mathfrak{s}$ is a split map. We define $\Phi: \mathbf{C} \rightarrow \mathbf{C}$ by $\Phi\left( \pm I_{2}\right)= \pm I_{2}$ and $\Phi( \pm S)=\mp S$. If $V \in \operatorname{Im} \mathfrak{s} \backslash\left\{I_{2}\right\}$, then $\widehat{V}=\Phi$ and $\widehat{V^{*}}=-\Phi$. Moreover, if $A$ is a the rotation of angle $\alpha$, we denote by $A^{\prime}$ the rotation of angle $\pi-\alpha$.

Proposition 10 Let $(U, C)$ be a $\mathfrak{s}$-pair associated to a common p-tuple of real planes. Then its orbit contains exactly two $\mathfrak{s}$-pairs. Moreover, the other $\mathfrak{s}$-pair $(\tilde{U}, \tilde{C})$ is related to $(U, C)$ according the following relations

$$
\begin{array}{ll}
\tilde{U}_{i j}=U_{i j}^{\prime}, & 1 \leq i \leq n, 2 \leq j \leq n, i \neq j, \quad(i, j) \neq(1,2) \\
\tilde{C}_{i j}=C_{i j}, & 3 \leq i \neq j \leq n \\
\tilde{C}_{2 j}=-C_{2 j}, & 3 \leq j \leq n .
\end{array}
$$

It follows that each common $\mathfrak{s}$-orbit contains exactly one $\mathfrak{s}$-pair such that $C_{23} \in$ $\left\{I_{2}, S\right\}$. This $\mathfrak{s}$-pair and the edge matrix fully characterized the p-tuple.

Proof. Apply the formulae (1) and (2) with $E=S$.

\subsubsection{Triangles}

A s-pair will depends on three rotations of $\operatorname{Im} \mathfrak{s}$ (the block-coefficients $U_{13}, U_{23}$ and $U_{32}$ ) and one element of $\mathbf{C}$ (the block-coefficient $C_{23}=C_{32}$ ). The s-orbit of the $\mathfrak{s}$-pair $(U, C)$ has at most two elements which are $(U, C)$ and $S \cdot(U, C)$. By a straightforward verification $(S \cdot C)_{23}=\varepsilon_{23} \varepsilon_{32} \varepsilon_{13} C_{23}$, where $\varepsilon_{i j}=1$ if $U_{i j}=I_{2}$ and -1 otherwise. It follows that, if the number of identity blocks in $U$ is even, there are exactly two elements in the orbit of $(U, C)$, and exactly one with $C_{23} \in\left\{I_{2}, S\right\}$. This $\mathfrak{s}$-pair is a numerical invariant which fully characterizes the $p$-tuple.

If some block $U_{i j}$ equals $I_{2}$, then $(S \cdot U)_{i j}=\mathfrak{s}\left(\widehat{U_{1 i}}(S)\right)=I_{2}$, and if some block $U_{i j}$ does not equal $I_{2},(S \cdot U)_{i j}=-U_{i j}^{*}$. It follows that there are exactly 
$16 \mathfrak{s}$-orbits which are singletons, those whose matrix $U$ contains only identity blocks, and those whose matrix $U$ contains one identity block and two blocks equal to $R\left(\frac{\pi}{2}\right)$. The other orbits have two elements. For $\alpha, \beta, \gamma \in\left[-\frac{\pi}{2}, \frac{\pi}{2}\right]$ and $E \in \mathbf{C}$, we denote by $O(E, \alpha, \beta, \gamma)$ the $\mathfrak{s}$-orbit of the $\mathfrak{s}$-pair

$$
\left.(U, C)=\left(\begin{array}{ccc}
I_{2} & I_{2} & R\left(\frac{\pi}{2}+\alpha\right) \\
I_{2} & I_{2} & R\left(\frac{\pi}{2}+\beta\right) \\
I_{2} & R\left(\frac{\pi}{2}+\gamma\right) & I_{2}
\end{array}\right),\left(\begin{array}{ccc}
I_{2} & I_{2} & I_{2} \\
I_{2} & I_{2} & E \\
I_{2} & E & I_{2}
\end{array}\right)\right) .
$$

Strictly speaking, if one of the angle $\alpha, \beta, \gamma$ is equal to $\frac{\pi}{2}$, then the above pair $(U, C)$ is not a s-pair. In this case, $O(E, \alpha, \beta, \gamma)$ should be defined as the orbit of a $\mathfrak{s}$-pair corresponding to the $\Delta$-Gram matrix $U * C * \Delta * U^{*}$, where $\Delta$ is an edge matrix small enough to ensure that $U * C * \Delta * U^{*}$ is positive semi-definite. We have the following equalities:

$$
\begin{aligned}
O(E, \alpha, \beta, \gamma) & =O(-E,-\alpha,-\beta,-\gamma) \\
O\left(E, \pm \frac{\pi}{2}, \beta, \gamma\right) & =O\left(E, \pm \frac{\pi}{2},-\beta,-\gamma\right) \\
O\left(E, \alpha, \pm \frac{\pi}{2}, \gamma\right) & =O\left(E,-\alpha, \pm \frac{\pi}{2},-\gamma\right) \\
O\left(E, \alpha, \beta, \pm \frac{\pi}{2}\right) & =O\left(E,-\alpha,-\beta, \pm \frac{\pi}{2}\right) .
\end{aligned}
$$

It follows that the space of orbits has two connected components ( $E= \pm I_{2}$ and $E= \pm S$ ); each of them is homeomorphic to a cube on which the points of each face are identified by the symmetry with respect to the center of the face.

Remark 11 A generic triple, as defined in [4], corresponds to an orbit $O( \pm E, \pm$ $\alpha, \pm \beta, \pm \gamma)$, with $0<|\alpha|,|\beta|,|\gamma|<\frac{\pi}{2}$. There is, in such an orbit, exactly one s-pair $(U, C)$ such that $C_{23}$ belongs to $\left\{I_{2}, S\right\}$. So the triple is characterized by its edge matrix, one sign $\varepsilon=\operatorname{det} C_{23}$ and the angles $\left.\alpha, \beta, \gamma \in\right] 0, \frac{\pi}{2}[$.

As stated in the introduction, Augustin Fruchard has proven that a generic triple is determined by its critical angles, its inner angles $\left.\omega_{1}, \omega_{2}, \omega_{3} \in\right] 0, \frac{\pi}{2}[$, and its signature $\left(\varepsilon_{1}, \varepsilon_{2}, \varepsilon_{3}, \varepsilon_{4}\right) \in\{ \pm 1\}^{4}$. Using the canonical form (see [4, p.130]), one can compute a Gram matrix from these invariants, and derive the $\mathfrak{s - p a i r . ~ A . ~ F r u c h a r d ' s ~ i n v a r i a n t s ~ a n d ~ o u r s ~ a r e ~ r e l a t e d ~ b y ~ t h e ~ f o l l o w i n g ~ f o r m u l a e ~}$

$$
\begin{aligned}
2 \alpha & =\left(2 \omega_{1}-\pi\right) \varepsilon_{1} \varepsilon_{2} \varepsilon_{3} \varepsilon_{4} \\
2 \beta & =\left(2 \omega_{2}-\pi\right) \varepsilon_{1} \varepsilon_{2} \varepsilon_{4} \\
2 \gamma & =\left(2 \omega_{3}-\pi\right) \varepsilon_{1} \varepsilon_{3} \\
\varepsilon & =\varepsilon_{1} \varepsilon_{2} .
\end{aligned}
$$

\subsubsection{Regular anisoclinic $p$-tuples in $G_{2}\left(\mathbb{R}^{2 p}\right)$}

From Proposition 7, a regular $p$-tuple is either common or special. The case of special $p$-tuples is settled by Theorem 3 and Theorem 6 . So we now consider the case of common regular $p$-tuples. In this case, we have necessarily $p=3$ or $p=4$, as stated by the 
Theorem 7 Let $\Gamma$ be an anisoclinic regular $p$-tuple of real planes. If $p>4$, then $\Gamma$ is special.

Proof. Let $\Gamma$ be regular, common, anisoclinic $p$-tuple of real planes with $p \geq 5$. Let $(U, C)$ be one of its two $\mathfrak{s}$-pairs. Since $\Gamma$ is common, the relation (18) becomes $\Phi\left(F_{k}\right) C_{2 k} F_{k}=-C_{2 k}(k=3, \ldots, p)$, whence $F_{k}= \pm S$; we can thus chose $F_{k}=S$. The formula (17) becomes $\mathfrak{s}\left(U_{i j}^{*}\right)=U_{i j}$; hence for $3 \leq i \neq j \leq p$, $U_{i j}=R\left(\frac{\pi}{2}\right)$ (the hypothesis $p>4$ is used here, for if $p \leq 4$, there is no triples of distinct indices $i, j, k \in\{3, \ldots, p\}$, and so, the formula (17) gives no relation). The relations (12) becomes $\mathfrak{s}\left(U_{2 j}^{*}\right)=U_{k j}$, whence $U_{2 j}=R\left(\frac{\pi}{2}\right)$. Put $\varepsilon=\operatorname{det} F_{1}$. The relation (3) yields $U_{1 j}=\varepsilon R\left(\varepsilon \frac{\pi}{2}\right)$. At last, (10) becomes $\mathfrak{s}\left(U_{1 j}^{*}\right)=\mathfrak{s}\left(U_{1 k}^{*} U_{1 j}\right)$, i.e. $R\left(\frac{\pi}{2}\right)=I_{2}$, which is absurd.

The case of triangles is given by the

Theorem 8 Let $\Gamma$ be an anisoclinic, regular, common triple of real planes. Then $\Gamma$ admits a unique $\mathfrak{s}$-pair which is either of the form

$$
\left(\left(\begin{array}{ccc}
I_{2} & I_{2} & A \\
I_{2} & I_{2} & A \\
I_{2} & A^{\prime} & I_{2}
\end{array}\right),\left(\begin{array}{ccc}
I_{2} & I_{2} & I_{2} \\
I_{2} & I_{2} & S \\
I_{2} & S & I_{2}
\end{array}\right)\right)
$$

or of the form

$$
\left(\left(\begin{array}{ccc}
I_{2} & I_{2} & A^{\prime} \\
I_{2} & I_{2} & A \\
I_{2} & A^{\prime} & I_{2}
\end{array}\right),\left(\begin{array}{ccc}
I_{2} & I_{2} & I_{2} \\
I_{2} & I_{2} & I_{2} \\
I_{2} & I_{2} & I_{2}
\end{array}\right)\right),
$$

where $A=R(\theta), 0<\theta<\pi$.

Remark 12 The Theorem 8 can be restated using the invariants $\alpha, \beta, \gamma, \varepsilon$ defined in Remark 11: a common triangle of planes is regular if and only if $-\alpha \varepsilon=\beta=-\gamma$. Each family of regular triangles corresponds to a diagonal of one the two cubic connected components of the space of $\mathfrak{s}$-orbits. The endpoints of these diagonals correspond to the special regular triangles.

Proof of Theorem 8. Let $(U, C)$ be the $\mathfrak{s - p a i r ~ a s s o c i a t e d ~ t o ~ a n ~ a n i s o c l i n i c ~}$ regular common triple of real planes, such that $E \stackrel{\text { def }}{=} C_{23} \in\left\{I_{2}, S\right\}$. Put $A=U_{23}, \varepsilon_{1}=\operatorname{det} F_{1}$ and $\varepsilon=\operatorname{det} E$. As seen in the proof of Theorem 7 , we can choose $F_{3}=S$ in the relations (3), ., (21). By straightforward computation, those of the relations $(3), \ldots,(21)$ which are not always satisfied become

$$
\begin{aligned}
A & =\mathfrak{s}\left(F_{1} U_{13}\right) \\
U_{13} & =\mathfrak{s}\left(F_{1} A\right) \\
\mathfrak{s}\left(E U_{32}^{*}\right) & =\mathfrak{s}\left(F_{1} U_{32}\right) \\
-\varepsilon & =\varepsilon_{1} \\
A^{\prime} & =U_{32} \\
U_{32}^{\prime} & =A,
\end{aligned}
$$


whence the conclusion.

Now let us examine the case of quadruples.

Theorem 9 Given two distinct angles $\alpha, \beta \in\left[0, \frac{\pi}{2}[\right.$, there exist at most four common regular quadruples of $G_{2}\left(\mathbb{R}^{8}\right)$ with critical angles $\alpha$ and $\beta$. They admit a $\mathfrak{s}$-pair which is either of the form

$$
\left(\left(\begin{array}{cccc}
I_{2} & I_{2} & A & A^{\prime} \\
I_{2} & I_{2} & A & A^{\prime} \\
I_{2} & A^{\prime} & I_{2} & A \\
I_{2} & A & A^{\prime} & I_{2}
\end{array}\right),\left(\begin{array}{cccc}
I_{2} & I_{2} & I_{2} & I_{2} \\
I_{2} & I_{2} & S & -S \\
I_{2} & S & I_{2} & \eta S \\
I_{2} & -S & \eta S & I_{2}
\end{array}\right)\right)
$$

or of the form

$$
\left(\left(\begin{array}{cccc}
I_{2} & I_{2} & A^{\prime} & A \\
I_{2} & I_{2} & A & A^{\prime} \\
I_{2} & A^{\prime} & I_{2} & A \\
I_{2} & A & A^{\prime} & I_{2}
\end{array}\right),\left(\begin{array}{cccc}
I_{2} & I_{2} & I_{2} & I_{2} \\
I_{2} & I_{2} & I_{2} & -I_{2} \\
I_{2} & I_{2} & I_{2} & -\eta I_{2} \\
I_{2} & -I_{2} & -\eta I_{2} & I_{2}
\end{array}\right)\right)
$$

where $\eta= \pm 1$ and $A=R\left(\frac{\pi}{2}-\eta \frac{\pi}{6}\right)$.

Proof. Let $(U, C)$ be the $\mathfrak{s}$-pair associated to an anisoclinic regular common quadruple of real planes, such that $C_{23} \in\left\{I_{2}, S\right\}$. Put $A=U_{23}$. As seen in the proof of Theorem 7 , we can choose $F_{k}=S$ for $k \geq 3$ in relations (3), $\ldots,(21)$. In particular, (11), (12) and (16) become respectively $U_{k 2}=U_{2 k}^{\prime}$ $(3 \leq k \leq p), U_{k j}=U_{2 j}^{\prime}(3 \leq j \neq k \leq p)$ and $U_{i k}^{\prime}=U_{i 2}(3 \leq i \neq k \leq p)$. Hence $U_{34}=U_{42}=A$ and $U_{24}=U_{33}=U_{43}=A^{\prime}$. Put $B \stackrel{\text { def }}{=} R(\theta) \stackrel{\text { def }}{=} U_{13}$, by (3), we have $U_{14}=B^{\prime}$ and either $B=A$ or $B=A^{\prime}$. With this notation, those of the relations $(3), \ldots,(21)$ which are not always satisfied become

$$
\begin{aligned}
A & =\mathfrak{s}\left(F_{1} B\right) \\
\mathfrak{s}\left(C_{23} A^{\prime *}\right) & =\mathfrak{s}\left(\Phi\left(F_{1}\right) A^{\prime}\right) \\
\mathfrak{s}\left(C_{24} A^{*}\right) & =\mathfrak{s}\left(\Phi\left(F_{1}\right) A\right) \\
\mathfrak{s}\left(C_{23} A^{\prime *} A\right) & =\mathfrak{s}\left(\Phi\left(F_{1}\right) A\right) \\
\mathfrak{s}\left(C_{24} A^{*} A^{\prime}\right) & =\mathfrak{s}\left(\Phi\left(F_{1}\right) A^{\prime}\right) \\
-\Phi\left(C_{23}\right) & =\varepsilon C_{23} \\
-\Phi\left(C_{24}\right) & =\varepsilon C_{24} \\
\widehat{A^{\prime *} A}\left(C_{23}\right) & =\widehat{A^{*} A^{\prime}}\left(C_{24}\right) \\
B^{\prime} & =\mathfrak{s}\left(B^{\prime *} B\right) \\
-C_{23} & =C_{43} \Phi\left(\delta\left(B^{\prime *} B\right)\right),
\end{aligned}
$$

where $\varepsilon=\operatorname{det} F_{1}$. From $(35)$, we get that $3 \theta=0(\bmod \pi)$, hence $\theta=\frac{\pi}{2}-\eta \frac{\pi}{6}$ for $\eta= \pm 1$. The angles of $A^{\prime *} A$ and $A^{*} A^{\prime}$ are opposite to each other and 
do not vanish, whence $\widehat{A^{\prime *} A}= \pm \Phi$ and $\widehat{A^{*} A^{\prime}}=\mp \Phi$ and thus (34) becomes $\Phi\left(C_{23}\right)=-\Phi\left(C_{24}\right)$, whence $C_{23}=-C_{24}$. Now we discuss two cases.

Case 1: $F_{1}=I_{2}$. By (27), $A=B$. The relations (32) implies $C_{23}= \pm S$. Since, by choice of the s-pair, $C_{23} \in\left\{S, I_{2}\right\}$, we have $C_{23}=S$ and $C_{24}=-S$. The equation (36) becomes $C_{34}=-\Phi\left(\delta\left(R\left(-\eta \frac{\pi}{2}\right)\right)\right) S=\eta S$. With these values of $C$ and $U$, all the relations (27), ., (36) are satisfied.

Case 2: $F_{1}=S$. By (27), $A=B^{\prime}$. The relations (32) implies $C_{23}= \pm I_{2}$. Since, by choice of the s-pair, $C_{23} \in\left\{S, I_{2}\right\}$, we have $C_{23}=I_{2}$ and $C_{24}=-I_{2}$. The equation (36) becomes $C_{34}=-\Phi\left(\delta\left(R\left(\eta \frac{\pi}{2}\right)\right)\right) I_{2}=-\eta I_{2}$. With these values of $C$ and $U$, all the relations $(27), \ldots,(36)$ are satisfied.

\subsection{Anisoclinic regular $p$-tuples of real 3-spaces.}

We recall that a rotation $R \in \mathbf{S O}(3)$ is associated to a pair of mutually opposite quaternions in the following way: if we identify $\mathbb{R}^{3}$ with the set of pure imaginary quaternions, the rotation of angle $\theta$ and unitary axis vector $u \in \mathbb{R}^{3}$ is represented by the unitary quaternions $\pm q= \pm\left(\cos \frac{\alpha}{2}+\sin \frac{\alpha}{2} u\right)$. Then, for any vector of $v \in \mathbb{R}^{3}$, i.e. for any pure imaginary quaternion $v$, we have $R(v)=q v \bar{q}$. In order to avoid confusion with indices, we denote by bold characters $(\mathbf{i}, \mathbf{j}$, $\mathbf{k})$ the vectors of the canonical basis of $\mathbb{R}^{3}$. Denote by $R_{\varepsilon_{0} \varepsilon_{1} \varepsilon_{2} \varepsilon_{3}}\left(\varepsilon_{0}, \varepsilon_{1}, \varepsilon_{2}\right.$, $\left.\varepsilon_{3} \in\{0, \pm 1\}\right)$ the set of those rotations which are associated to a quaternion number $q=a_{0}+a_{1} \mathbf{i}+a_{2} \mathbf{j}+a_{3} \mathbf{k}$, such that $\varepsilon_{i}=\operatorname{sgn}\left(a_{i}\right)(i=0, \ldots, 3)$. Obviously $R_{-\varepsilon_{0}-\varepsilon_{1}-\varepsilon_{3}-\varepsilon_{3}}=R_{\varepsilon_{0} \varepsilon_{1} \varepsilon_{3} \varepsilon_{3}}$ and the 40 elements set

$$
P \stackrel{\text { def }}{=}\left\{R_{\varepsilon_{0} \varepsilon_{1} \varepsilon_{3} \varepsilon_{3}} \mid \varepsilon_{0}, \varepsilon_{1}, \varepsilon_{2}, \varepsilon_{3}=-1,0,1\right\}
$$

is a partition of $\mathbf{S O}(3)$. In order to shorten the notation, we shall only write the $\operatorname{sign}(+$ or -$)$ when one of the indices $\varepsilon_{k}$ equals \pm 1 . We also define $R_{z}(z=0$, $1,2,3)$ as the union of those $R_{\varepsilon_{0} \varepsilon_{1} \varepsilon_{3} \varepsilon_{3}}$ such that exactly $z$ indices among $\varepsilon_{0}$, $\ldots, \varepsilon_{3}$ equal zero. Clearly

$$
Q \stackrel{\text { def }}{=}\left\{R_{k} \mid k=0, \ldots 3\right\}
$$

is also a partition of $S U(3)$, coarser that $P$.

In the case of anisoclinic tuples of real 3 -spaces, $\mathbf{C}= \pm\left\{I_{3}, S_{\mathbf{i}}, S_{\mathbf{j}}, S_{\mathbf{k}}\right\}$, where $S_{q}(q=\mathbf{i}, \mathbf{j}, \mathbf{k})$ is the half-turn of axis $q$, or in other words, the rotation associated to the quaternion $q$. It is easy to check that the right or left multiplication by $S_{q}(q=\mathbf{i}, \mathbf{j}, \mathbf{k})$ respects the partition $P$ of $\mathbf{S O}(3)$. More precisely we have

$$
\begin{array}{lll}
S_{\mathbf{i}} R_{\varepsilon_{0} \varepsilon_{1} \varepsilon_{2} \varepsilon_{4}}=R_{-\varepsilon_{1}} \varepsilon_{0}-\varepsilon_{3} \varepsilon_{2} & R_{\varepsilon_{0} \varepsilon_{1} \varepsilon_{2} \varepsilon_{4}} S_{\mathbf{i}}=R_{-\varepsilon_{1}} \varepsilon_{0} \varepsilon_{3}-\varepsilon_{2} \\
S_{\mathbf{j}} R_{\varepsilon_{0} \varepsilon_{1} \varepsilon_{2} \varepsilon_{4}}=R_{-\varepsilon_{2}} \varepsilon_{3} \varepsilon_{0}-\varepsilon_{1} & R_{\varepsilon_{0} \varepsilon_{1} \varepsilon_{2} \varepsilon_{4}} S_{\mathbf{j}}=R_{-\varepsilon_{2}}-\varepsilon_{3} \varepsilon_{0} \varepsilon_{1} \\
S_{\mathbf{k}} R_{\varepsilon_{0} \varepsilon_{1} \varepsilon_{2} \varepsilon_{4}}=R_{-\varepsilon_{3}}-\varepsilon_{2} \varepsilon_{1} \varepsilon_{0} & R_{\varepsilon_{0} \varepsilon_{1} \varepsilon_{2} \varepsilon_{4}} S_{\mathbf{k}}=R_{-\varepsilon_{3}} \varepsilon_{2}-\varepsilon_{1} \varepsilon_{0} .
\end{array}
$$

In particular each set $R_{z} \in Q$ is globally invariant under multiplications by elements of $\mathbf{C}^{+} \stackrel{\text { def }}{=} \mathbf{C} \cap \mathbf{S O}(3)$. Now let us examine the left cosets. If $U$ belongs to some $R_{\varepsilon} \in P\left(\varepsilon \in\{ \pm 1,0\}^{4}\right)$, then the three other elements of the coset $U \mathbf{C}$, 
lie in three elements of $P$ that does not depends on $U \in R_{\varepsilon}$. Using (37), we get the following table.

\begin{tabular}{|l||l|l|l|l|l|l|l|}
\hline$R_{\varepsilon}$ & $R_{+000}$ & $R_{++00}$ & $R_{+0+0}$ & $R_{+00+}$ & $R_{0-++}$ \\
$R_{\varepsilon} S_{\mathbf{i}}$ & $R_{0+00}$ & $R_{+-00}$ & $R_{0+0-}$ & $R_{0++0}$ & $R_{+0+-}$ \\
$R_{\varepsilon} S_{\mathbf{j}}$ & $R_{00+0}$ & $R_{00++}$ & $R_{+0-0}$ & $R_{0-+0}$ & $R_{++0+}$ \\
$R_{\varepsilon} S_{\mathbf{k}}$ & $R_{000+}$ & $R_{00-+}$ & $R_{0+0+}$ & $R_{+00-}$ & $R_{+--0}$ \\
\hline \\
$\qquad$\begin{tabular}{ll|l|l|l|l|}
$R_{0+-+}$ & $R_{0++-}$ & $R_{0+++}$ & $R_{+---}$ & $R_{++++}$ \\
$R_{+0--}$ & $R_{+0++}$ & $R_{+0-+}$ & $R_{++-+}$ & $R_{+--+}$ \\
$R_{+-0+}$ & $R_{+-0-}$ & $R_{++0-}$ & $R_{+++-}$ & $R_{++--}$ \\
$R_{+++0}$ & $R_{++-0}$ & $R_{+-+0}$ & $R_{+-++}$ & $R_{+-+-}$ \\
\hline
\end{tabular}
\end{tabular}

Hence, each coset $U \mathbf{C}$ contains exactly one element lying in one of the ten sets of the first line of the above table. By definition $\mathfrak{s}(U)$ is this element. If $U \in \mathbf{C}$, i.e. , if either $U$ or $-U$ belongs to one of the sets of the first column of the table, then $\mathfrak{s}(U)$ belongs to $R_{+000}=\left\{I_{3}\right\}$. Hence $\mathfrak{s}$ is a split map. It is now easy to compute the maps $\widehat{U}$, which depends only of the set $R_{\varepsilon} \in P$ in which lies $U$. We obtain

$$
\begin{aligned}
& \text { if } U \in R_{+000} \text {, then } \widehat{U}=\widehat{U^{*}}=\operatorname{id} \mathbf{C}, \\
& \text { if } U \in R_{++00} \text {, then } \widehat{U}=\tau_{\mathbf{j k}} \text { and } \widehat{U^{*}}=\tau_{\mathbf{i}} \text {, } \\
& \text { if } U \in R_{+0+0} \text {, then } \widehat{U}=\tau_{\mathbf{i k}} \text { and } \widehat{U^{*}}=\tau_{\mathbf{j}} \text {, } \\
& \text { if } U \in R_{+00+} \text {, then } \widehat{U}=\tau_{\mathbf{i j}} \text { and } \widehat{U^{*}}=\tau_{\mathbf{k}} \text {, } \\
& \text { if } U \in R_{1} \cap \operatorname{Im} \mathfrak{s} \text {, then } \widehat{U}=\widehat{U^{*}}=\mathrm{id} \mathbf{C}, \\
& \text { if } U \in R_{+---} \text {, then } \widehat{U}=\sigma \text { and } \widehat{U^{*}}=\sigma^{-1}, \\
& \text { if } U \in R_{++++} \text {, then } \widehat{U}=\sigma^{-1} \text { and } \widehat{U^{*}}=\sigma,
\end{aligned}
$$

where $\tau_{q q^{\prime}}\left(q, q^{\prime}=\mathbf{i}, \mathbf{j}, \mathbf{k}\right)$ exchanges $\pm S_{q}$ and $\pm S_{q^{\prime}}$ and fixes the other elements of $\mathbf{C}, \tau_{q}$ exchanges $\pm S_{q}$ and $\pm I_{3}$ and fixes the other elements of $\mathbf{C}$, and $\sigma$ is defined by

$$
\begin{array}{cc}
\sigma\left( \pm I_{3}\right)= \pm I_{3} & \sigma\left( \pm S_{\mathbf{i}}\right)= \pm S_{\mathbf{j}} \\
\sigma\left( \pm S_{\mathbf{j}}\right)= \pm S_{\mathbf{k}} & \sigma\left( \pm S_{\mathbf{k}}\right)= \pm S_{\mathbf{i}}
\end{array}
$$

Using this split map, one can obtain some results analogous to section 3.1.1, namely, can determine the topology of the space of anisoclinic triangles. However, this would involve to discuss a wild number of cases, and we choose to not include such a technical piece of work in the present article.

Therefore, we shall restrict our study to regular $p$-tuples. The first step of this study is the following refinement of Proposition 7.

Proposition 13 Let $(U, C)$ be a $\mathfrak{s}$-pair associated to an anisoclinic p-tuples of real 3-spaces. Then there exists $z=0,1,2,3$ such that for all indices $i, j$ $(1 \leq i \leq p, 2 \leq j \leq p, i \neq j,(i, j) \neq(1,2)), U_{i j}$ belongs to $R_{z}$. Moreover, this number $z$ is the same for all the $\mathfrak{s}$-pair of a given $\mathfrak{s}$-orbit. 
Proof. The statement follows from relations (3), (11), (12), (15) and (1), and from the fact that $R_{z}$ is stable by multiplication by any element of $\mathbf{C}$, as well as by $\mathfrak{s}$.

The number $z$ defined in the above Proposition will be called the degree of the regular $p$-tuple. The case of special $p$-tuples (i.e. of degree 3 ) is treated in section 2 , so we consider now the case of common ones.

Theorem 10 There is no anisoclinic regular triangle of 3-spaces of degree 0.

Proof. Let $(U, C)$ be a $\mathfrak{s}$-pair associated to an anisoclinic regular triangle of 3spaces of degree 0 . The matrix $U_{13}$ belongs to $R_{0}$, i.e. there exists $\varepsilon= \pm 1$ such that $U_{13} \in R_{+\varepsilon \varepsilon \varepsilon}$. The relation (9) yields $\mathfrak{s}\left(F_{3} U_{13}\right)=\mathfrak{s}\left(U_{13}^{*}\right)$. On the one hand, a direct computation shows that, for any $E \in \mathbf{C}, R_{+\varepsilon \varepsilon \varepsilon}$ is stable by the map $U \longmapsto \mathfrak{s}(E U)$, whence $\mathfrak{s}\left(U_{13}^{*}\right) \in R_{+\varepsilon \varepsilon \varepsilon}$. On the other hand $U_{13}^{*} \in R_{-\varepsilon \varepsilon \varepsilon} \subset \operatorname{Im} \mathfrak{s}$, whence $\mathfrak{s}\left(U_{13}^{*}\right)=U_{13}^{*} \in R_{-\varepsilon \varepsilon \varepsilon}$.

Theorem 11 An anisoclinic regular triangle of 3-spaces of degree 1 admits a unique $\mathfrak{s}$-pair of the form

$$
\left(\left(\begin{array}{ccc}
I_{3} & I_{3} & F_{1} A F_{1} \\
I_{3} & I_{3} & A \\
I_{3} & A & I_{3}
\end{array}\right),\left(\begin{array}{ccc}
I_{3} & I_{3} & I_{3} \\
I_{3} & I_{3} & \varepsilon F_{1} \\
I_{3} & \varepsilon F_{1} & I_{3}
\end{array}\right)\right)
$$

where $A \in R_{0+++}, F_{1} \in \mathbf{C}^{+}$and $\varepsilon= \pm 1$.

Proof. Let $R_{\varepsilon}$ be one of the sets $R_{0+--}, R_{0+-+}, R_{0++-}$ or $R_{0+++}$. A straightforward verification shows that $\mathfrak{s}\left(E R_{\varepsilon}\right)$ equals successively each of the above sets when $E$ run through $\mathbf{C}^{+}$. It follows that, a $\mathfrak{s - o r b i t ~ a s s o c i a t e d ~ t o ~ a n ~ a n i s o c l i n i c ~}$ regular $p$-tuple of 3 -spaces of degree 1 contains exactly four $\mathfrak{s}$-pairs, and exactly one of them such that $U_{23} \in R_{0+++}$. Let $(U, C)$ be this $\mathfrak{s}$-pair and put $A \stackrel{\text { def }}{=} U_{23}$. By (3), there exists $F_{1} \in \mathbf{C}^{+}$such that $U_{13}=F_{1} A F_{1}$. For $k=3$, the equation (9) becomes $F_{3} U_{13} F_{3}=U_{13}$, so the axis of $U_{13} \in R_{1}$ is invariant under $F_{3}$. It follows that $F_{3}=I_{3}$. Now, (13) implies $U_{32}=U_{23}$. For $j=3$, the equation (5) becomes $C_{23} A C_{23}=F_{1} A F_{1}$, whence the axis of $A$ is invariant by $C_{23} F_{1}$, and so $C_{23}=F_{1}$.

Conversely, a $\mathfrak{s}$-pair of the above form satisfies all the formulae $(3), \ldots,(21)$.

\section{Theorem 12}

i. For $p>4$, there is no anisoclinic regular $p$-tuples of degree 1 in $G_{3}\left(\mathbb{R}^{3 p}\right)$.

ii. An anisoclinic regular quadruple of 3-spaces of degree 1 admits a $\mathfrak{5}$-pair of the form

$$
\left(\left(\begin{array}{cccc}
I_{3} & I_{3} & F_{1} A F_{1} & F_{1} B F_{1} \\
I_{3} & I_{3} & A & B \\
I_{3} & A & I_{3} & B \\
I_{3} & B & A & I_{3}
\end{array}\right),\left(\begin{array}{cccc}
I_{3} & I_{3} & I_{3} & I_{3} \\
I_{3} & I_{3} & \varepsilon F_{1} & \varepsilon F_{1} \\
I_{3} & \varepsilon F_{1} & I_{3} & \varepsilon F_{1} S_{q} \\
I_{3} & \varepsilon F_{1} & \varepsilon F_{1} S_{q} & I_{3}
\end{array}\right)\right),
$$


where $A \in R_{0+++}$ is a half-turn whose axis makes an angle $\frac{\pi}{3}$ with one vector $q \in\{\mathbf{i}, \mathbf{j}, \mathbf{k}\}, B=S_{q} A S_{q}, F_{1} \in \mathbf{C}^{+} \backslash\left\{S_{q}\right\}$.

Proof. As seen in the proof of Theorem 11, a s-orbit associated to an anisoclinic regular $p$-tuple of 3 -spaces of degree 1 contains exactly four $\mathfrak{s}$-pairs, and exactly one of them such that $U_{23} \in R_{0+++}$. Let $(U, C)$ be this $\mathfrak{s}$-pair and put $A \stackrel{\text { def }}{=} U_{23}$. Since all non-identity block-coefficients of $U$ are half-turns, the relation (9) becomes $\mathfrak{s}\left(F_{k} U_{1 k}\right)=U_{1 k}$. Moreover $\mathfrak{s}\left(F_{k} U_{1 k}\right)=F_{k} U_{1 k} \widehat{U_{1 k}}\left(F_{k}\right)=F_{k} U_{1 k} F_{k}$, whence $F_{k}$ preserves the axis of $U_{1 k}$, and so $F_{k}=I_{3}(k \geq 3)$. Now the relations (11) and (12) give $U_{2 k}=U_{k 2}$ and $U_{k j}=U_{2 j}$. The relation (5) yields $C_{2 j} U_{j 2} C_{2 j}=F_{1} U_{j 2} F_{1}$, whence there exist $\varepsilon_{3}, \ldots, \varepsilon_{p}= \pm 1$ such that $C_{2 j}=\varepsilon_{j} F_{1}$. The relation (8) becomes

$$
\varepsilon_{i} \varepsilon_{j} \widehat{U_{2 i} U_{2 j}}\left(F_{1}\right) C_{i j} \widehat{U_{2 j} U_{2 i}}\left(F_{1}\right)=C_{i j} .
$$

Since $\operatorname{Im} \mathfrak{s} \subset \mathbf{S O}(3)$, we have the formula $\operatorname{det}(\widehat{V}(E))=\operatorname{det} V \operatorname{det} E$. Hence, (38) infers that $\varepsilon_{i}$ does not depends on $i=3, \ldots, p$ and that $F_{i j} \stackrel{\text { def }}{=} \widehat{U_{2 i} U_{2 j}}\left(F_{1}\right)=$ $F_{j i}$. The formula (3) shows that $U_{1 i}=F_{1} U_{2 i} F_{1}$.

Assume now that $p \geq 5$. For any pair of distinct indices $i, k \in\{1, \ldots, p\}$, there exists an index $j \in\{1, \ldots, p\}$ distinct from $i$ and $j$. Hence the relation (17) gives $U_{i j}=\delta\left(U_{1 k} U_{1 i}\right) U_{i j} \delta\left(U_{1 k} U_{1 i}\right)$. It follows that the axis of $U_{i j} \in R_{1}$ is stable under $\delta\left(U_{1 k} U_{1 i}\right) \in \mathbf{C}^{+}$, whence $\mathfrak{s}\left(U_{1 k} U_{1 i}\right)=U_{1 k} U_{1 i}$. Now (10) becomes $U_{1 j}=U_{1 k} U_{1 j}$, in contradiction with the fact that $U_{1 k} \in R_{1}$.

Put $B \stackrel{\text { def }}{=} U_{24}$. For $(k, i)=(3,4)$, the equation (14) becomes

$$
\begin{aligned}
B & =\mathfrak{s}\left(\delta\left(F_{1} A B F_{1}\right) A\right) \\
& =\mathfrak{s}\left(F_{34} F_{1} A\right)=F_{34} F_{1} A F_{34} F_{1} .
\end{aligned}
$$

Hence $B$ and $A$ are conjugated by some matrix of $\mathbf{C}$. The equation (6) gives $\mathfrak{s}\left(F_{1} A B\right)=\mathfrak{s}\left(F_{1} B\right)$, i.e. $A B F_{34}=B F_{1}$. Moreover $A \neq B$ follows from (6). Combining this equation and (39) gives $\left(F_{34} F_{1} A\right)^{3}=I_{3}$. It follows that $F_{34} F_{1} A$ is a rotation of angle $2 \pi / 3$, and that $F_{34} F_{1} \neq I_{3}$. Hence there exists $q \in\{\mathbf{i}, \mathbf{j}, \mathbf{k}\}$ such that $F_{1} F_{34}=S_{q}$. Since the angle of $S_{q} A$ is $2 \pi / 3$, the angle between the axis of $A$ and $q$ is $\pi / 3$. It remains to prove that $C_{34}=\varepsilon F_{34}$; this follows from equation (20).

Conversely, one can check that a $\mathfrak{s}$-pair of the above form satisfies all the equations $(3), \ldots,(21)$.

From now on, if $V$ if a rotation of angle $\alpha$, we shall denote by $V^{\prime}$ the rotation with the same axis and angle $\pi-\alpha$. A direct computation shows that, if $V \in R_{++00}$ and $F \in \mathbf{C}^{+}$, then $\mathfrak{s}(F V)$ is either $V$ (if $F=I_{3}$ or $F=S_{\mathbf{i}}$ ) or $V^{\prime}=S_{\mathbf{i}} V^{*}=V^{*} S_{\mathbf{i}}$ (if $F=S_{\mathbf{j}}$ or $F=S_{\mathbf{k}}$ ).

\section{Theorem 13}

$i$. There is no anisoclinic regular quintuples of 3-spaces of degree 2 . 
ii. An anisoclinic regular quadruple of $G_{3}\left(\mathbb{R}^{12}\right)$ admits a $\mathfrak{5}$-pair which is either of the form

$$
\begin{aligned}
& \left(\left(\begin{array}{cccc}
I_{3} & I_{3} & A & A^{\prime} \\
I_{3} & I_{3} & A & A^{\prime} \\
I_{3} & A^{\prime} & I_{3} & A \\
I_{3} & A & A^{\prime} & I_{3}
\end{array}\right)\right. \\
& \left.\left(\begin{array}{cccc}
I_{3} & I_{3} & I_{3} & I_{3} \\
I_{3} & I_{3} & \varepsilon \sigma\left(S_{q}\right) & \varepsilon \sigma^{-1}\left(S_{q}\right) \\
I_{3} & \varepsilon \sigma\left(S_{q}\right) & I_{3} & \varepsilon \sigma\left(S_{q}\right) S_{q}^{(\eta-1) / 2} \\
I_{3} & \varepsilon \sigma^{-1}\left(S_{q}\right) & \varepsilon \sigma\left(S_{q}\right) S_{q}^{(\eta-1) / 2} & I_{3}
\end{array}\right)\right),
\end{aligned}
$$

or of the form

$$
\left(\left(\begin{array}{cccc}
I_{3} & I_{3} & A^{\prime} & A \\
I_{3} & I_{3} & A & A^{\prime} \\
I_{3} & A^{\prime} & I_{3} & A \\
I_{3} & A & A^{\prime} & I_{3}
\end{array}\right),\left(\begin{array}{cccc}
I_{3} & I_{3} & I_{3} & I_{3} \\
I_{3} & I_{3} & \varepsilon I_{2} & \varepsilon S_{q} \\
I_{3} & \varepsilon I_{2} & I_{3} & \varepsilon S_{q}^{(\eta+1) / 2} \\
I_{3} & \varepsilon S_{q} & \varepsilon S_{q}^{(\eta+1) / 2} & I_{3}
\end{array}\right)\right),
$$

where $q \in\{\mathbf{i}, \mathbf{j}, \mathbf{k}\}, \eta, \varepsilon= \pm 1$, and $A$ is a rotation of axis $q$ and angle $\frac{\pi}{2}-\eta \frac{\pi}{6}$. Moreover this $\mathfrak{s}$-pair is unique.

Proof. Let $(\tilde{U}, \tilde{C})$ be a s-pair associated to such a $p$-tuple $(p \geq 4)$. Since $(\tilde{U}, \tilde{C})$ is of degree two, $\tilde{U}_{23}$ belongs to one of the three sets $R_{++00}, R_{+0+0}$ or $R_{+00+}$. We assume here that $\tilde{U}_{23} \in R_{++00}$; one can easily check that the proofs in the two other cases are similar. By $(2)$, we have $\left(S_{\mathbf{j}} \cdot \tilde{C}\right)_{23}=S_{\mathbf{i}} \tilde{C}_{23}$. Hence, there is in the $\mathfrak{s}$-orbit of $(\tilde{U}, \tilde{C})$ a $\mathfrak{s}$-pair $(U, C)$ such that $E \stackrel{\text { def }}{=} C_{23} \in\left\{ \pm I_{3}, \pm S_{\mathbf{j}}\right\}$. Moreover, since $S_{\mathbf{i}} \cdot(\tilde{U}, \tilde{C})=(\tilde{U}, \tilde{C})$ and $S_{\mathbf{j}} \cdot(\tilde{U}, \tilde{C})=S_{\mathbf{k}} \cdot(\tilde{U}, \tilde{C})$, this $\mathfrak{5}$-pair is unique.

By virtue of (4), (11), (12) and (16), each block $U_{i j}(1 \leq i \leq p, 2 \leq j \leq p$, $i \neq j,(i, j) \neq(1,2))$ equal either $A \stackrel{\text { def }}{=} U_{23}$ or $A^{\prime}$. Assume that there exists $k \geq 3$ such that $F_{k}=I_{3}$ or $F_{k}=S_{\mathbf{i}}$, then by (9), $U_{1 k}=U_{1 k}^{\prime}$, and so all the non-identity block-coefficients of $U$ are all equal to a same rotation of angle $\pi / 2$. In this case the relation (6) becomes $I_{3}=\mathfrak{s}\left(\tau_{\mathbf{j k}}\left(F_{1}\right) U_{i j}\right)$, which is absurd. Hence, for $k \geq 3, F_{k}$ equals either $S_{\mathbf{k}}$ or $S_{\mathbf{j}}$.

If $p \geq 5$, then for $(i, j, k)=(3,4,5)$, the relation (17) becomes $U_{34}^{\prime}=$ $\mathfrak{s}\left(\delta\left(U_{15}^{*} U_{13}\right) U_{34}\right)$. Since $U_{15}^{*}$ and $U_{13}$ are rotation of axis $\mathbf{i}, \delta\left(U_{15}^{*} U_{13}\right) \in\left\{I_{3}, S_{\mathbf{i}}\right\}$, whence $U_{34}^{\prime}=U_{34}$. This would imply once again that all the non-identity blocks of $U$ are equal, in contradiction with (6). If follows that $p=4$.

It follows from (11) that $U_{32}=A^{\prime}$. For $(i, k)=(3,4)$, the relation (16) gives $U_{34}^{\prime}=A$. By (12) (with $(j, k)=(4,3)$ ), we have $U_{24}=A^{\prime}$. By (4) we have either $U_{13}=A$ and $U_{14}=A^{\prime}$, or $U_{13}=A^{\prime}$ and $U_{14}=A$. In both cases $U_{13}=U_{14}^{\prime}$. For $(j, k)=(3,4)$ the relation (10) gives $U_{13}^{\prime}=\mathfrak{s}\left(U_{13}^{\prime *} U_{13}\right)$. Hence the angle of $U_{13}$ is either $\pi / 3$ or $2 \pi / 3$. It follows that there exists $\eta= \pm 1$ such that $A$ is the rotation of axis $\mathbf{i}$ and angle $\frac{\pi}{2}-\eta \frac{\pi}{6}$. Now we discuss two cases. 
Case 1: $F_{1} \in\left\{I_{3}, S_{\mathbf{i}}\right\}$. In this case $U_{13}=A$ and $U_{14}=A^{\prime}$. The relation (7) becomes $\tau_{\mathbf{i}}\left(C_{2 j}\right)=C_{2 j}$, whence $C_{23}=\varepsilon S_{\mathbf{j}}$ for $\varepsilon= \pm 1$. For $(j, k)=(3,4),(19)$ becomes $S_{\mathbf{i}} C_{23}=C_{43} \tau_{\mathbf{j k}}\left(\delta\left(A^{\prime *} A\right)\right)$, thus $C_{43}=\varepsilon S_{\mathbf{j}} S_{\mathbf{i}}^{(\eta-1) / 2}$. For $(j, k)=(4,3)$ the same relation becomes $S_{\mathbf{i}} C_{24}=C_{43} \tau_{\mathbf{j k}}\left(\delta\left(A^{*} A^{\prime}\right)\right)$, whence $C_{24}=\varepsilon S_{\mathbf{k}}$.

Case 2: $F_{1} \in\left\{S_{\mathbf{j}}, S_{\mathbf{k}}\right\}$. In this case $U_{13}=A^{\prime}$ and $U_{14}=A$. The relation (7) becomes $\tau_{\mathbf{i}}\left(C_{2 j}\right)=S_{\mathbf{i}} C_{2 j}$, whence $C_{23}=\varepsilon I_{3}$ for $\varepsilon= \pm 1$. For $(j, k)=(3,4),(19)$ becomes $S_{\mathbf{i}} C_{23}=C_{43} \tau_{\mathbf{j k}}\left(\delta\left(A^{*} A^{\prime}\right)\right)$, thus $C_{43}=\varepsilon S_{\mathbf{i}}^{(\eta+1) / 2}$. For $(j, k)=(4,3)$ the same relation becomes $S_{\mathbf{i}} C_{24}=C_{43} \tau_{\mathbf{j k}}\left(\delta\left(A^{\prime *} A\right)\right)$, whence $C_{24}=\varepsilon S_{\mathbf{i}}$.

So the s-pair $(U, C)$ is necessarily of one of the two forms given in the statement of the Theorem. It is now straightforward to check that these $\mathfrak{s}$-pairs are actually regular.

Theorem 14 An anisoclinic regular triangle of 3-spaces of degree 2 admits a unique $\mathfrak{s}$-pair $(U, C)$ which is either of the form

$$
\left(\left(\begin{array}{ccc}
I_{3} & I_{3} & A \\
I_{3} & I_{3} & A \\
I_{3} & A^{\prime} & I_{3}
\end{array}\right),\left(\begin{array}{ccc}
I_{3} & I_{3} & I_{3} \\
I_{3} & I_{3} & \pm \sigma\left(S_{q}\right) \\
I_{3} & \pm \sigma\left(S_{q}\right) & I_{3}
\end{array}\right)\right),
$$

or of the form

$$
\left(\left(\begin{array}{ccc}
I_{3} & I_{3} & A^{\prime} \\
I_{3} & I_{3} & A \\
I_{3} & A^{\prime} & I_{3}
\end{array}\right),\left(\begin{array}{ccc}
I_{3} & I_{3} & I_{3} \\
I_{3} & I_{3} & \pm I_{3} \\
I_{3} & \pm I_{3} & I_{3}
\end{array}\right)\right)
$$

where $A \in R_{2} \cap \operatorname{Im} \mathfrak{s}$ is a rotation of axis $q \in\{\mathbf{i}, \mathbf{j}, \mathbf{k}\}$.

Proof. Let $(\tilde{U}, \tilde{C})$ be a s-pair associated to an anisoclinic regular triangle of 3 -spaces of degree 2 . We assume for the proof that $A \stackrel{\text { def }}{=} U_{23} \in R_{++00}$; the two other cases are similar. As seen in the proof of Theorem 13, there is in the s-orbit of $(\tilde{U}, \tilde{C})$ a unique $\mathfrak{s}$-pair $(U, C)$ such that $E \stackrel{\text { def }}{=} C_{23} \in\left\{ \pm I_{3}, \pm S_{\mathbf{j}}\right\}$.

The relation (18) gives $\tau_{\mathbf{j k}}\left(F_{3}\right) F_{3} E=E S_{\mathbf{i}}$, whence $F_{3} \in\left\{S_{\mathbf{j}}, S_{\mathbf{k}}\right\}$. Hence, by (11), we have $U_{32}=A^{\prime}$. We now discuss two cases.

Case 1: $F_{1} \in\left\{I_{3}, S_{\mathbf{i}}\right\}$. The relation (4) implies that $U_{13}=A$. The relation (5) gives $\mathfrak{s}(E A)=A^{\prime}$, and so $E= \pm S_{\mathbf{j}}$. It is a straightforward verification to check that $(U, C)$ satisfies all the equation $(3), \ldots,(21)$.

Case 2: $F_{1} \in\left\{S_{\mathbf{j}}, S_{\mathbf{k}}\right\}$. The relation (4) implies that $U_{13}=A^{\prime}$. The relation (5) gives $\mathfrak{s}(E A)=A$, and so $E= \pm I_{3}$ It is a straightforward verification to check that $(U, C)$ satisfies all the equation $(3), \ldots,(21)$.

\section{Anisoclinic $p$-tuple of $\mathbb{C}$-spaces}

\subsection{The split map}

In this section $\mathbb{K}=\mathbb{C}$ and $n=2$. For $\rho \in\left[0, \frac{\pi}{2}\right]$, and $\alpha, \beta \in \mathbb{R} / 2 \pi \mathbb{Z}$, we put

$$
v_{\rho}(\alpha, \beta)=\left(\begin{array}{cc}
\cos \rho e^{i \alpha} & -\sin \rho e^{-i \beta} \\
\sin \rho e^{i \beta} & \cos \rho e^{-i \alpha}
\end{array}\right) .
$$


It is well-known that $\mathbf{S U}(2)$ is the set of those matrices $v_{\rho}(\alpha, \beta)$. Therefore, each unitary matrix $V$ admits a decomposition of the form $V=z v_{\rho}(\alpha, \beta)$, with $z \in \mathbb{U}$, where $\mathbb{U}$ stands for the unitary circle in $\mathbb{C}$. Indeed, $V$ admits exactly two decompositions of this form, for $z v_{\rho}(\alpha, \beta)=(-z) v_{\rho}(\alpha+\pi, \beta+\pi)$.

We shall use the following notation

$$
\begin{aligned}
v_{\rho}(\alpha) & =v_{\rho}(\alpha, 0) \\
c(\theta) & =v_{0}(\theta, 0) \\
d(\beta) & =v_{\frac{\pi}{2}}(0, \beta) .
\end{aligned}
$$

We shall consider here anisoclinic $p$-tuples of complex planes, thus the group $\mathbf{C}$ is the group of unitary diagonal matrix:

$$
\mathbf{C}=\{z c(\alpha) \mid z \in \mathbb{U}, \alpha \in \mathbb{R} / 2 \pi \mathbb{Z}\} .
$$

Elementary calculus gives the following formulae

$$
\begin{aligned}
& c(\theta) v_{\rho}(\alpha, \beta)=v_{\rho}(\alpha+\theta, \beta-\theta) \\
& v_{\rho}(\alpha, \beta) c(\theta)=v_{\rho}(\alpha+\theta, \beta+\theta) .
\end{aligned}
$$

If follows that, if $\rho \in] 0, \frac{\pi}{2}\left[\right.$, there is exactly one element of the form $v_{\rho}(\phi)$ in the left coset

$$
v_{\rho}(\alpha, \beta) \mathbf{C}=\left\{z v_{\rho}(\alpha+\theta, \beta+\theta) \mid \theta \in \mathbb{R}, z \in \mathbb{U}\right\},
$$

namely $v_{\rho}(\alpha-\beta)$. For $\rho=\frac{\pi}{2}$, the left coset $d(\beta) \mathbf{C}$ is the set

$$
\{z d(\beta) \mid \beta \in \mathbb{R}, z \in \mathbb{U}\} .
$$

It follows that the map $\mathfrak{s}$ defined by

$$
\begin{aligned}
\mathfrak{s}\left(z v_{\rho}(\alpha, \beta)\right) & =v_{\rho}(\alpha-\beta), & & z \in \mathbb{U}, \rho \in] 0, \frac{\pi}{2}[, \alpha, \beta \in \mathbb{R} / 2 \mathbb{Z}, \\
\mathfrak{s}(z d(\beta)) & =d(0), & & z \in \mathbb{U}, \beta \in \mathbb{R}, \\
\mathfrak{s}(E) & =I_{2}, & E \in \mathbf{C}, &
\end{aligned}
$$

is a split map. The associated map $\delta$ is given by the formulae

$$
\begin{aligned}
\delta\left(v_{\rho}(\alpha, \beta)\right) & =c(\beta) \\
\delta(d(\beta)) & =c(\beta),
\end{aligned}
$$

and the maps $\widehat{V}$ and $\widehat{V^{*}}$, for $V \in \operatorname{Im} \mathfrak{s}$ are given by

$$
\begin{aligned}
\widehat{v_{\rho}(\alpha)}(c(\theta)) & =c(-\theta) & & (\rho \in] 0, \frac{\pi}{2}[, \alpha, \theta, \in \mathbb{R}) \\
\widehat{v_{\rho}(\alpha)^{*}}(c(\theta)) & =-c(-\theta) & & (\rho \in] 0, \frac{\pi}{2}[, \alpha, \theta, \in \mathbb{R}) \\
\widehat{d(0)}(c(\theta)) & =c(-\theta) & & \\
\widehat{d(0)^{*}}(c(\theta)) & =-c(-\theta) . & &
\end{aligned}
$$

As explained in the Remark 5, the $\mathfrak{s}$-orbits under $\mathbf{C}$ are indeed the orbits under $\mathbf{C}^{+} \stackrel{\text { def }}{=}\{c(\theta) \mid \theta \in \mathbb{R}\}$. 


\subsection{Anisoclinic triangles of $G_{2}\left(\mathbb{C}^{6}\right)$}

Consider a triangle of $G_{2}\left(\mathbb{C}^{6}\right)$ associated to a $\mathfrak{s}$-pair $(U, C)$ such that

$$
U=\left(\begin{array}{ccc}
I_{2} & I_{2} & v_{\rho_{1}}\left(\alpha_{1}\right) \\
I_{2} & I_{2} & v_{\rho_{2}}\left(\alpha_{2}\right) \\
I_{2} & v_{\rho_{3}}\left(\alpha_{3}\right) & I_{2}
\end{array}\right)
$$

It follows from (1) and from the formulae of the above Section that the numbers $\rho_{i}$ that appear in the above matrix depend only of the $\mathfrak{s}$-orbit. These numbers are invariants of the triangle, which are indeed closely related to inner angles. We say that an anisoclinic triangle is generic if these three numbers $\rho_{1}, \rho_{2}, \rho_{3}$ belong to $] 0, \frac{\pi}{2}[$. This definition generalizes A. Fruchard's one. It is quite clear that the set of generic triangles is open and dense in the set of all triangles.

Proposition 14 Each generic triangle admits a single $\mathfrak{s}$-pair of the form

$$
\left.\left(\begin{array}{ccc}
I_{2} & I_{2} & v_{\rho_{1}}\left(\alpha_{1}\right) \\
I_{2} & I_{2} & v_{\rho_{2}}\left(\alpha_{2}\right) \\
I_{2} & v_{\rho_{3}}\left(\alpha_{3}\right) & I_{2}
\end{array}\right),\left(\begin{array}{ccc}
I_{2} & I_{2} & I_{2} \\
I_{2} & I_{2} & \omega I_{2} \\
I_{2} & \bar{\omega} I_{2} & I_{2}
\end{array}\right)\right),
$$

where $\left.\rho_{1}, \rho_{2}, \rho_{3} \in\right] 0, \frac{\pi}{2}\left[, \alpha_{1}, \alpha_{2}, \alpha_{3} \in \mathbb{R} / 2 \pi \mathbb{Z}, \omega \in \mathbb{U}\right.$. Therefore, the set of $\mathfrak{s - o r b i t s ~ o f ~ g e n e r i c ~ t r i a n g l e s ~ o f ~} G_{2}\left(\mathbb{C}^{6}\right)$ is homeomorphic to $] 0,1\left[^{3} \times \mathbb{U}^{4}\right.$.

Proof. The result follows from the formula

$$
\begin{gathered}
c(\theta / 2) \cdot\left(\left(\begin{array}{ccc}
I_{2} & I_{2} & v_{\rho_{1}}\left(\alpha_{1}\right) \\
I_{2} & I_{2} & v_{\rho_{2}}\left(\alpha_{2}\right) \\
I_{2} & v_{\rho_{3}}\left(\alpha_{3}\right) & I_{2}
\end{array}\right),\left(\begin{array}{ccc}
I_{2} & I_{2} & I_{2} \\
I_{2} & I_{2} & \omega c(\phi) \\
I_{2} & \bar{\omega} c(-\phi) & I_{2}
\end{array}\right)\right)= \\
\left(\left(\begin{array}{ccc}
I_{2} & I_{2} & v_{\rho_{1}}\left(\alpha_{1}+\theta\right) \\
I_{2} & I_{2} & v_{\rho_{2}}\left(\alpha_{2}+\theta\right) \\
I_{2} & v_{\rho_{3}}\left(\alpha_{3}-\theta\right) & I_{2}
\end{array}\right),\left(\begin{array}{ccc}
I_{2} & I_{2} & I_{2} \\
I_{2} & I_{2} & \omega c(\phi-\theta) \\
I_{2} & \bar{\omega} c(-\phi+\theta) & I_{2}
\end{array}\right)\right),
\end{gathered}
$$

which in turn, follows from (1), (2) and the formulae of Section 4.1.

\subsection{Regular $p$-tuples in $G_{2}\left(\mathbb{C}^{d}\right)$}

Proposition 15 Let $(U, C)$ be a $\mathfrak{s}$-pair associated to a common anisoclinic $p$ tuple of complex 3-spaces. Then, either all or none of the $U_{i j}(1 \leq i \leq p$, $2 \leq j \leq p, i \neq j,(i, j) \neq(1,2)$ equal $d(0)$.

Proof. The statement follows from relations (3), (11), (12) and (15) and from the fact that $\mathfrak{s}(E d(0))=d(0)$ for any $E \in \mathbf{C}$.

Theorem 15 A common anisoclinic triangle of $G_{2}\left(\mathbb{C}^{6}\right)$ is regular if and only if it admits a $\mathfrak{s}$-pair which is of the form

$$
\left(\left(\begin{array}{ccc}
I_{2} & I_{2} & v_{\rho}(0) \\
I_{2} & I_{2} & v_{\rho}(0) \\
I_{2} & v_{\rho}(\pi) & I_{2}
\end{array}\right),\left(\begin{array}{ccc}
I_{2} & I_{2} & I_{2} \\
I_{2} & I_{2} & \eta S \\
I_{2} & \eta S & I_{2}
\end{array}\right)\right)
$$


or of the form

$$
\left(\left(\begin{array}{ccc}
I_{2} & I_{2} & v_{\rho}(\pi) \\
I_{2} & I_{2} & v_{\rho}(0) \\
I_{2} & v_{\rho}(\pi) & I_{2}
\end{array}\right),\left(\begin{array}{ccc}
I_{2} & I_{2} & I_{2} \\
I_{2} & I_{2} & \eta I_{2} \\
I_{2} & \eta I_{2} & I_{2}
\end{array}\right)\right)
$$

or of the form

$$
\left.\left(\begin{array}{ccc}
I_{2} & I_{2} & d(0) \\
I_{2} & I_{2} & d(0) \\
I_{2} & d(0) & I_{2}
\end{array}\right),\left(\begin{array}{ccc}
I_{2} & I_{2} & I_{2} \\
I_{2} & I_{2} & \omega I \\
I_{2} & \bar{\omega} I & I_{2}
\end{array}\right)\right),
$$

where $\rho \in] 0, \frac{\pi}{2}[, \eta= \pm 1, \omega \in\{1, i\}$. Moreover, this $\mathfrak{s - p a i r ~ i s ~ u n i q u e . ~}$

Remark 16 It follows from Theorem 15 that the regular triangles of $G_{3}\left(\mathbb{C}^{6}\right)$ are isometric to regular triangles of $G_{2}\left(\mathbb{R}^{6}\right)$. The correspondence between the above $\mathfrak{s}$-pairs and the regular orbits described in section 3.1.2 is the following: for $\alpha \neq 0, O(S, \alpha, \alpha,-\alpha)$ corresponds to a $\mathfrak{s}$-pair of the first form, and $O\left(I_{2},-\alpha, \alpha,-\alpha\right)$ corresponds to a s-pair of the second form. The correspondence between $(\eta, \rho)$ and $\alpha$ is given by $\rho=\frac{\pi}{2}-|\alpha|$ and $\eta=-\operatorname{sgn}(\alpha)$. The $\mathfrak{s - o r b i t s} O\left(I_{2}, 0,0,0\right)$ and $O(S, 0,0,0)$ correspond to $\mathfrak{s}$-pairs of the third form, with $\omega=1$ and $\omega=i$ respectively.

Proof of Theorem 15. Let $(\tilde{U}, \tilde{C})$ be a $\mathfrak{s - p a i r ~ a s s o c i a t e d ~ t o ~ a ~ c o m m o n ~ a n i s o - ~}$ clinic regular triangle. From Proposition 15, either all the non-identity blocks of $\tilde{U}$ or none of them equal $d(0)$. Assume first that we are in the second case. Let $\omega$ be the square root of $\operatorname{det}\left(\tilde{C}_{23}\right)$ whose argument belongs to $[0, \pi[$. There exists $\phi \in \mathbb{R}$ such that $\tilde{C}_{23}=\omega c(\phi)$. By $(2),(c(\phi / 2) \cdot \tilde{C})_{23}=\omega I_{2}$. Therefore, there exists in the same orbit a s-pair $(U, C)$ such that $C_{23}=\omega I_{2}$. Put $F_{1}=c\left(\theta_{1} / 2\right)$. The s-pair $(U, C)$ satisfies the relation $(7)$, which becomes $\bar{\omega} c(-\phi+\pi)=\omega F_{1}^{* 2}$, whence $\omega^{2} I_{2}=c\left(\pi+\theta_{1}\right) \in \mathbf{C}^{+}$. It follows that $\omega^{2}= \pm 1$ and $\omega \in\{1, i\}$. Conversely, given any numbers $\phi \in \mathbb{R}, \omega \in\{1, i\}$, there exists $\theta_{1} \in \mathbb{R}$ such that the relation is satisfied. All other relations become $d(0)=d(0)$, except (18) which becomes, for $F_{3}=c\left(\theta_{3}\right), \omega c\left(\phi-2 \theta_{3}\right)=\bar{\omega} c(\pi-\phi)$. Obviously, one can find $\theta_{3}$ such that this last relation is satisfied.

Now assume that $\tilde{U}_{13}, \tilde{U}_{23}$, and $\tilde{U}_{32}$ are not $d(0)$. Put $\tilde{U}_{23}=v_{\rho}\left(\alpha_{2}\right)$. By (1), $\left(c\left(-\alpha_{2} / 2\right) \cdot \tilde{U}\right)_{23}=v_{\rho}(0)$. So there exists in the same orbit a unique $\mathfrak{s}^{-}$ pair $(U, C)$ such that $U_{23}=v_{\rho}(0)$. Since the triangle is regular, there exists $F_{1} \stackrel{\text { def }}{=} c\left(\theta_{1} / 2\right)$ and $F_{3} \stackrel{\text { def }}{=} c\left(\theta_{3} / 2\right)$ such that the relations $(3), \ldots,(21)$ are satisfied. Put $U_{13}=v_{\rho_{1}}\left(\alpha_{1}\right)$ and $U_{32}=v_{\rho_{3}}\left(\alpha_{3}\right)$. By the relation (11) becomes $v_{\rho}\left(\theta_{3}\right)=v_{\rho_{3}}\left(\alpha_{3}\right)$, whence $\rho_{3}=\rho$ and $\theta_{3}=\alpha_{3}$. The relations (3) and (4), become respectively $v_{\rho}(0)=v_{\rho_{1}}\left(\alpha_{1}+\theta_{1}\right)$ and $v_{\rho_{1}}\left(\alpha_{1}\right)=v_{\rho}\left(\theta_{1}\right)$. It follows that $\rho_{1}=\rho$ and $\theta_{1}=\alpha_{1} \in\{0, \pi\}$. The relation (9) becomes $v_{\rho}\left(\alpha_{1}+\theta_{3}\right)=$ $v_{\rho}\left(-\alpha_{1}-\pi\right)$, whence $\theta_{3}=\pi$. Put $C_{23}=\omega c(\phi)$, the relations $(7)$ and (18) becomes $c\left(\theta_{1}\right) \omega^{2}=-I_{2}$ and $c(2 \phi) \omega^{2}=I_{2}$. So $(U, C)$ is necessarily one of the two first $\mathfrak{s}$-pairs of the Theorem. It is now straightforward to check that these $\mathfrak{s - p a i r s ~ a c t u a l l y ~ s a t i s f i e d ~ a l l ~ t h e ~ r e l a t i o n s ~}(3), \ldots,(21)$. 


\section{Theorem 16}

i. There is no anisoclinic common regular quintuple in $G_{2}\left(\mathbb{C}^{10}\right)$.

ii. An anisoclinic common quadruple of $G_{2}\left(\mathbb{C}^{8}\right)$ is regular if and only if it admits a $\mathfrak{s}$-pair of the form

$$
\left(\left(\begin{array}{cccc}
I_{2} & I_{2} & v_{\frac{\pi}{3}}(0) & v_{\frac{\pi}{3}}(\pi) \\
I_{2} & I_{2} & v_{\frac{\pi}{3}}(0) & v_{\frac{\pi}{3}}(\pi) \\
I_{2} & v_{\frac{\pi}{3}}(\pi) & I_{2} & v_{\frac{\pi}{3}}(0) \\
I_{2} & v_{\frac{\pi}{3}}(0) & v_{\frac{\pi}{3}}(\pi) & I_{2}
\end{array}\right),\left(\begin{array}{cccc}
I_{2} & I_{2} & I_{2} & I_{2} \\
I_{2} & I_{2} & \varepsilon S & -\varepsilon S \\
I_{2} & \varepsilon S & I_{2} & \varepsilon S \\
I_{2} & -\varepsilon S & \varepsilon S & I_{2}
\end{array}\right)\right)
$$

or of the form

$$
\left(\left(\begin{array}{cccc}
I_{2} & I_{2} & v_{\frac{\pi}{3}}(\pi) & v_{\frac{\pi}{3}}(0) \\
I_{2} & I_{2} & v_{\frac{\pi}{3}}(0) & v_{\frac{\pi}{3}}(\pi) \\
I_{2} & v_{\frac{\pi}{3}}(\pi) & I_{2} & v_{\frac{\pi}{3}}(0) \\
I_{2} & v_{\frac{\pi}{3}}(0) & v_{\frac{\pi}{3}}(\pi) & I_{2}
\end{array}\right),\left(\begin{array}{cccc}
I_{2} & I_{2} & I_{2} & I_{2} \\
I_{2} & I_{2} & \varepsilon I_{2} & -\varepsilon I_{2} \\
I_{2} & \varepsilon I_{2} & I_{2} & -\varepsilon I_{2} \\
I_{2} & -\varepsilon I_{2} & -\varepsilon I_{2} & I_{2}
\end{array}\right)\right),
$$

where $\varepsilon= \pm 1$. Moreover, this $\mathfrak{s}$-pair is unique.

Remark 17 As in the case of triangles, the common regular quadruples are isometric to real ones. The above $\mathfrak{s}$-pairs and those of Theorem 9 are corresponding first form to first form, and second to second, with $\eta=\varepsilon$.

Proof of Theorem 16. Let $(U, C)$ be a $\mathfrak{s}$-pair associated to an anisoclinic common regular $p$-tuple $(p \geq 4)$. By Proposition 15, either all of the $U_{i j}(1 \leq$ $i \leq p, 2 \leq j \leq p, i \neq j,(i, j) \neq(1,2))$ or none of them equal $d(0)$. In the latter case, the relation (6) implies $I_{2}=d(0)$. Hence this case is not possible.

So there exists some numbers $\left.\rho_{i, j} \in\right] 0, \frac{\pi}{2}\left[\right.$ and $\alpha_{i j} \in \mathbb{R} / 2 \pi \mathbb{Z}$ such that $U_{i j}=v_{\rho_{i j}}\left(\alpha_{i j}\right)(1 \leq i \leq p, 2 \leq j \leq p, i \neq j,(i, j) \neq(1,2))$. As seen in the proof of Theorem 15, there is in the $\mathfrak{s}$-orbit of $(U, C)$ an only $\mathfrak{s}$-pair such that $\alpha_{23}=0$; we assume that $(U, C)$ is this one. From (3), (11), (12) and (14) it follows that $\rho_{i j}$ does not depends on $i, j$; we simply denote by $\rho$ the common value. Put $F_{i}=c\left(\theta_{i} / 2\right)$; the relations (3) and (4) become $v_{\rho}\left(\alpha_{2 j}\right)=v_{\rho}\left(\alpha_{1 j}+\theta_{1}\right)$ and $v_{\rho}\left(\alpha_{1 j}\right)=v_{\rho}\left(\alpha_{2 j}+\theta_{1}\right)$, whence $\alpha_{2 j}=\alpha_{1 j}+\theta_{1}$ and either $\theta_{1}=0$ or $\theta_{1}=\pi$. The relation (9) gives for $k \geq 3, v_{\rho}\left(\alpha_{2 k}+\theta_{k}+\theta_{1}\right)=v_{\rho}\left(\pi-\alpha_{2 k}+\theta_{1}\right)$, therefore $\theta_{k}=\pi-2 \alpha_{2 k}$. The relation (10) becomes $v_{\rho}\left(\alpha_{2 j}+\pi-2 \alpha_{2 k}+\theta_{1}\right)=$ $\mathfrak{s}\left(v_{\rho}\left(-\alpha_{2 k}+\theta_{1}, \pi\right) v_{\rho}\left(\alpha_{2 j}+\theta_{1}\right)\right)$. Thus, there exists $z \in \mathbb{U}$ such that

$$
v_{\rho}\left(\alpha_{2 j}+\pi-2 \alpha_{2 k}+\theta_{1}\right)=v_{\rho}\left(-\alpha_{2 k}+\theta_{1}, \pi\right) v_{\rho}\left(\alpha_{2 j}+\theta_{1}\right) \operatorname{diag}(z, \bar{z}) .
$$

Computing the matrix product and equaling the three last coefficients give

$$
\begin{aligned}
\bar{z} e^{i \theta_{1}}\left(e^{-i \alpha_{2 k}}-e^{-i \alpha_{2 j}}\right) \cos \rho-1 & =0 \\
z \cos \rho e^{-i \theta_{1}}\left(e^{i \alpha_{2 j}}-e^{i \alpha_{2 k}}\right)+1 & =0 \\
-e^{-i\left(\alpha_{2 j}-2 \alpha_{2 k}-\theta_{1}\right)} \cos \rho-\bar{z}\left(e^{-i\left(\alpha_{2 j}-\alpha_{2 k}\right)} \cos ^{2} \rho+\sin ^{2} \rho\right) & =0 .
\end{aligned}
$$


The second equation shows that $\alpha_{2 j} \neq \alpha_{2 k}$. If we use it to compute $z$, and then replace it in the two other ones we get

$$
\begin{aligned}
&\left(e^{i \alpha_{2 k}}-e^{i \alpha_{2 j}}\right)^{2} \cos ^{2} \rho+e^{i\left(\alpha_{2 j}+\alpha_{2 k}\right)}=0 \\
&\left(e^{i \alpha_{2 j}}-e^{i \alpha_{2 k}}\right)\left(e^{i\left(\alpha_{2 k}-\alpha_{2 j}\right)} \cos ^{2} \rho+\sin ^{2} \rho\right)-e^{i\left(2 \alpha_{2 k}-\alpha_{2 j}\right)}=0 .
\end{aligned}
$$

Eliminating $\rho$ in these equations gives $e^{2 i \alpha_{k}}=e^{2 i \alpha_{j}}$, whence $\alpha_{k}=\alpha_{j}+\pi$ for $j \neq k \in\{3, \ldots, p\}$. It follows that $p=4$ and $\alpha_{24}=\alpha_{23}+\pi=\pi$, and thus, $\theta_{3}=\theta_{4}=\pi$. Now, for $(k, j)=(3,4),(40)$ becomes

$$
4 \cos ^{2} \rho=1,
$$

and implies $\rho=\frac{\pi}{3}$. The relation (12) becomes $v_{\rho}\left(\alpha_{2 j}+\theta_{k}\right)=v_{\rho}\left(\alpha_{k, j}\right)$, thus $\alpha_{k j}=\alpha_{2 j}+\pi(k, j=3,4, k \neq j)$. At last, the relation (11) gives $\alpha_{k 2}=\alpha_{2 k}+\pi$. Hence the matrix $U$ must be of one of the two forms given in the Theorem.

Put $C_{i j}=\omega_{i j} c\left(\phi_{i j}\right)$ with $\omega_{i j} \in \mathbb{U}$ and $\phi_{i j} \in[0, \pi[$. The relation (5) becomes

$$
v_{\frac{\pi}{3}}\left(-\alpha_{j 2}+2 \phi_{2 i}\right)=v_{\frac{\pi}{3}}\left(\alpha_{2 j}+\pi+\theta_{1}\right),
$$

whence $\phi_{23}=\phi_{24}=\frac{\theta_{1}+\pi}{2}(\bmod \pi)$. From now on, we distinguish two cases:

Case 1: $\theta_{1}=0$ and $\phi_{23}=\phi_{24}=\frac{\pi}{2}$. For $j=3$ the relation (7) gives $\omega_{23}^{2}=-1$, hence there exists $\varepsilon= \pm 1$ such that $\omega_{23}=-\varepsilon i$. For $(j, k)=(4,3)$, the relation (20) becomes $\omega_{23} \omega_{34} I_{2}=c\left(\phi_{43}-\frac{\pi}{2}\right)$. Hence $c\left(\phi_{43}-\frac{\pi}{2}\right)$ is a scalar matrix, $\phi_{34}=\frac{\pi}{2}$ and $\omega_{34}=\omega_{23}=-\varepsilon i$. For $(j, k)=(4,3)$, the same relation gives $\omega_{24}=-\omega_{34}=\varepsilon i$. Finally

$$
\begin{aligned}
& C_{23}=C_{34}=-\varepsilon i c\left(\frac{\pi}{2}\right)=\varepsilon S \\
& C_{24}=\varepsilon i c\left(\frac{\pi}{2}\right)=-\varepsilon S .
\end{aligned}
$$

Case 2: $\theta_{1}=\pi$ and $\phi_{23}=\phi_{24}=0$. For $j=3$ the relation (7) gives $\omega_{23}^{2}=1$, hence $\varepsilon \stackrel{\text { def }}{=} \omega_{23}= \pm 1$. For $(j, k)=(3,4)$, the relation $(20)$ becomes $\omega_{23} \omega_{34} I_{2}=$ $-c\left(\phi_{43}\right)$. Hence $c\left(\phi_{43}\right)$ is a scalar matrix, $\phi_{34}=0$ and $\omega_{34}=-\omega_{23}=-\varepsilon$. For $(j, k)=(4,3)$ the same relation gives $\omega_{24}=\omega_{34}=-\varepsilon$.

It follows that a $\mathfrak{s}$-pair associated to a regular quadruple lies necessarily in one of the four $\mathfrak{s}$-orbits described in the Theorem. On the other hand, by Theorem 9 , there are at least four regular $\mathfrak{s - o r b i t s . ~ T h u s , ~ w e ~ d o ~ n o t ~ e v e n ~ h a v e ~}$ to check that all the relations $(3), \ldots,(21)$ are satisfied.

\section{Miscellaneous comments}

\section{$5.1 \quad$ On special $p$-tuples}

Although special $p$-tuples are defined by mean of their $\mathfrak{s}$-pairs, the notion actually does not depends on the choice of the split map. This is clear from the 
fact that all split maps are asked to map the group $\mathbf{C}$ on the identity matrix. It follows that specialness carries some geometrical meaning. In the case of an anisoclinic $p$-tuples of $G_{2}\left(\mathbb{R}^{d}\right)$, special $p$-tuples are simply those for which all the inner angles equal either 0 or $\pi / 2$. This could be generalized for any homogeneous $p$-tuple $\Gamma=\left(\Gamma_{1}, \ldots, \Gamma_{p}\right)$ in the following way. Projecting the unit sphere of $\Gamma_{i}$ onto $\Gamma_{k}$ yields an ellipsoid $\mathcal{E}_{i}^{k} \subset \Gamma_{k}$. The $p$-tuple is special if and only if, for each $n$-space $\Gamma_{k}$ of the $p$-tuple, there exist $(n-1)(n-2)$ linear maps $f_{i j}^{k}$ of $\Gamma_{k}$ which are all diagonal in the same basis of $\Gamma_{k}$, and such that $f_{i j}^{k}\left(\mathcal{E}_{i}^{k}\right)=\mathcal{E}_{j}^{k}$. If the main axes of the ellipsoids are well defined, (i.e. in the anisoclinic case), it is equivalent to state that all the ellipsoids of a given $n$-spaces share the same axes.

In the three cases we have studied $\left(G_{2}\left(\mathbb{R}^{d}\right), G_{3}\left(\mathbb{R}^{d}\right)\right.$ and $\left.G_{2}\left(\mathbb{C}^{d}\right)\right)$, it appears that a regular quintuple is always special. Whether this fact holds with a greater degree of generality is an important open question.

\subsection{On the (equi-)isoclinic case}

If the $p$-tuple is isoclinic, then $\mathbf{C}=\mathbf{U}(n)$ and $\mathfrak{s}=1$. It follows that each $\mathfrak{s}$ pair is special. The group $\mathbf{C}$ act on the second matrix of $\mathfrak{s}$-pairs according the formula

$$
(E \cdot C)_{i j}=E C_{i j} E^{*}
$$

So the situation is analogous to the equi-isoclinic one. An isoclinic $p$-tuple is determined by the critical angles (which are $\frac{p(p-1)}{2}$ numbers) and by the orbit of its normal Seidel matrix. The only difference is that we cannot compute this matrix from the Gram matrix with a global formula, but need to define it blockwise.

We have not studied in this paper the isoclinic regular $p$-tuples, for this has already be done, at least in the cases of $G_{2}\left(\mathbb{R}^{d}\right)[2]$ and $G_{3}\left(\mathbb{R}^{d}\right)[3]$. We just recall some of the results of these two papers in order to emphasize the differences between isoclinic and anisoclinic case.

First of all, an equi-isoclinic triangle of $G_{n}\left(\mathbb{R}^{3 n}\right)$ is always regular. This fact could be, of course, derived from Theorem 2, had it not already been proven in [2]. It follows that the set of (regular) $\mathfrak{s}$-orbits of equi-isoclinic triangles is homeomorphic to the set of conjugation classes of the orthogonal group of degree $n$. If $n=2$, it is homeomorphic to the disjoint union of the segment $[0, \pi]$ (where $x \in[0, \pi]$ corresponds to the class $\{R(x), R(-x)\}$ ) and a singleton (the class of conjugation of all the symmetries).

Doing the same job in the complex case gives the following result: an equiisoclinic triangle with $\mathfrak{s}$-pair $\left(\left[I_{n}\right]_{3}, C\right)$ is regular if and only if $C_{23}$ and $C_{23}^{*}$ are conjugated. Hence, some non-regular equi-isoclinic triangles exist in $G_{n}\left(\mathbb{C}^{3 n}\right)$. The dimension (over $\mathbb{R}$ ) of the space of $\mathfrak{s}$-orbits of equi-isoclinic triangles is $n$, while the dimension (over $\mathbb{R}$ ) of the space of regular $\mathfrak{s}$-orbits is only $\left\lfloor\frac{n}{2}\right\rfloor$. Knowing that the matrices of $\mathbf{U}(n)$ which are conjugated to their conjugate transpose are exactly those which are conjugated to some real matrix of $\mathbf{O}(n)$ leads to the following 
Proposition 18 An isoclinic regular triangle of $G_{n}\left(\mathbb{C}^{3 n}\right)$ is associated to a s-pair $\left(\left[I_{2}\right]_{3}, C\right)$ such that $C_{23} \in \mathbf{O}(n)$.

For $p \geq 4$, the regularity of a real $p$-tuple is no longer systematic, and indeed, occurs rather rarely. It is known that, a s-pair $\left(\left[I_{n}\right]_{p}, C\right)$ such that all the coefficient blocks $C_{i j}(2 \leq i \neq j \neq p)$ are equal to a symmetric matrix of $\mathbf{O}(n)$ is regular (Proposition 2.12 in [2]). If an isoclinic regular $\mathfrak{s}$-orbit does not contain one $\mathfrak{s}$-pair (or, equivalently, only $\mathfrak{s}$-pairs) of this form, we shall call it exceptional. For $n=2,3$, the complete list of regular isoclinic $\mathfrak{s}$-orbit is known (Theorem 6.2 in [2] for $n=2$, and Theorem 9 in [3] for $n=3$ ). It appears that, in both cases, there is no exceptional $\mathfrak{s}$-orbit for $p>5$. In the case of planes, the set of exceptional $\mathfrak{s}$-orbits is finite, while in the case of 3 -spaces, there exists two continuous families of $\mathfrak{s}$-orbits of quadruples.

\subsection{Isoclinic limit of anisoclinic $p$-tuples}

It is clear, from Theorem 4 , that for a given anisoclinic $\mathfrak{s}$-pair $(U, C)$, there exists some isoclinic $p$-tuple in the closure of the set of anisoclinic $p$-tuple associated to $(U, C)$. If $(U, C)$ is regular, then the corresponding $p$-tuple is clearly regular too. It follows that there exists a canonical map $\Lambda$ from the set of regular anisoclinic $\mathfrak{s}$-orbits to the set of isoclinic ones. If a regular isoclinic $\mathfrak{s}$-orbit does not belong to $\operatorname{Im} \Lambda$, we say that it is rigid. The intuitive meaning is that the corresponding $p$-tuples cannot be deformed into an anisoclinic one, without breaking the regularity. It is a natural problem to determine the list of rigid $\mathfrak{s}$-orbits. For $\mathbb{K}=\mathbb{R}$ and $n=2$ or $n=3$, the answers follows from [2], [3] and the present article. We present here the sole results. The proofs are straightforward, but, in some case, involve unpleasant computations.

First, we can see that the non-exceptional regular $\mathfrak{s}$-orbits are the images by $\Lambda$ of the special regular $\mathfrak{s}$-orbits. So only exceptional $\mathfrak{s}$-orbits may be rigid.

Second, no (regular) triangles of $G_{2}\left(\mathbb{R}^{6}\right)$ or $G_{3}\left(\mathbb{R}^{9}\right)$ is rigid. Indeed, if we denote by $O\left(V_{1}, V_{2}, V_{3}, E\right)$ the anisoclinic $\mathfrak{s}$-orbit of the s-pair $(U, C)$ such that $\left(V_{1}, V_{2}, V_{3}, E\right)=\left(U_{13}, U_{23}, U_{32}, C_{23}\right)$, and by $O(V)$ the isoclinic $\mathfrak{s}$-orbit of the s-pair $\left(\left[I_{n}\right]_{3}, C^{\prime}\right)$ such that $C_{23}^{\prime}=V$, then we have

$$
\Lambda \circ O\left(R(\pi-t), R(t), R(\pi-t), I_{2}\right)=O(R(3 t)) .
$$

Since the orbit $O(S)$ is not exceptional, the case of triangles of $G_{2}\left(\mathbb{R}^{6}\right)$ is settled. Concerning $G_{3}\left(\mathbb{R}^{9}\right)$, if we denote by $R_{\mathbf{i}}(\alpha)$ the rotation of angle $\alpha$ and axis $\mathbf{i}$, we have for $\varepsilon= \pm 1$

$$
\Lambda \circ O\left(R_{\mathbf{i}}(\pi-t), R_{\mathbf{i}}(t), R_{\mathbf{i}}(\pi-t), \varepsilon I_{2}\right)=O\left(\varepsilon R_{\mathbf{i}}(3 t)\right),
$$

and so, no triangles of $G_{3}\left(\mathbb{R}^{9}\right)$ is rigid.

It remains the case of quadruples. The four $\mathfrak{s}$-orbits of anisoclinic regular quadruples of $G_{2}\left(\mathbb{R}^{8}\right)$ are given by Theorem 9 . The image by $\Lambda$ of the two first 
of these $\mathfrak{s}$-orbits are the isoclinic $\mathfrak{s}$-orbit of

$$
\left(\left[I_{2}\right]_{4},\left(\begin{array}{cccc}
I_{2} & I_{2} & I_{2} & I_{2} \\
I_{2} & I_{2} & \eta I_{2} & \eta I_{2} \\
I_{2} & \eta I_{2} & I_{2} & \eta I_{2} \\
I_{2} & \eta I_{2} & \eta I_{2} & I_{2}
\end{array}\right)\right)
$$

where $\eta= \pm 1$ is the parameter used in Theorem 9. The two last one have the same image by $\Lambda$ which is the orbit of

$$
\left(\left[I_{2}\right]_{4},\left(\begin{array}{cccc}
I_{2} & I_{2} & I_{2} & I_{2} \\
I_{2} & I_{2} & R\left(-\frac{2 \pi}{3}\right) S & S \\
I_{2} & R\left(-\frac{2 \pi}{3}\right) S & I_{2} & R\left(\frac{2 \pi}{3}\right) S \\
I_{2} & S & R\left(\frac{2 \pi}{3}\right) S & I_{2}
\end{array}\right)\right)
$$

It follows that, among the five existing regular isoclinic $\mathfrak{s}$-orbits which are listed in Theorem 6.1 of [2], only the third and fourth are rigid.

The anisoclinic regular $\mathfrak{s}$-orbits of quadruples of $G_{2}\left(\mathbb{R}^{8}\right)$ are given by Theorems 12 and 13 . We denote by $O(\alpha)(\alpha \in] 0, \frac{\pi}{2}[)$ the anisoclinic orbit of quadruple of $G_{3}\left(\mathbb{R}^{12}\right)$ described in Theorem 12 , when $q=\mathbf{i}, \varepsilon=1, F_{1}=S_{\mathbf{k}}$, and $A$ is the half-turn of axis $\left(\frac{1}{2}, \frac{\sqrt{2}}{2} \cos \alpha, \frac{\sqrt{2}}{2} \sin \alpha\right)$. Then $\Lambda(O(\alpha))$ is an $\mathfrak{s}$ orbit of the continuous family described in Theorem 24 of [3]. The relation between $\alpha$ and the parameters $\kappa$ and $\theta$ of [3] are the following:

$$
\begin{aligned}
64 \cos \omega & =27 \cos 6 \alpha-54 \cos 4 \alpha-27 \cos 2 \alpha-10 \\
\kappa & =\operatorname{sgn}\left(\alpha-\cos ^{-1} \frac{\sqrt{3}}{3}\right) .
\end{aligned}
$$

Of course, if $\varepsilon=-1$, we obtain the corresponding indirect quadruple. It follows that none of the quadruples of the continuous family of [3] is rigid, except possibly those corresponding to $\omega=\pi$, i.e. $\alpha \in\left\{0, \frac{\pi}{2}\right\}$, that is, those given by the s-pairs

$$
\begin{gathered}
p_{0} \stackrel{\text { def }}{=}\left(\left[I_{2}\right]_{4},\left(\begin{array}{cccc}
I_{2} & I_{2} & I_{2} & I_{2} \\
I_{2} & I_{2} & \varepsilon S_{\mathbf{i}} & \varepsilon S_{\mathbf{i}} \\
I_{2} & \varepsilon S_{\mathbf{i}} & I_{2} & \varepsilon S_{\mathbf{i}} \\
I_{2} & \varepsilon S_{\mathbf{i}} & \pm S_{\mathbf{i}} & I_{2}
\end{array}\right)\right), \\
p_{\pi / 2} \stackrel{\text { def }}{=}\left(\left[I_{2}\right]_{4},\left(\begin{array}{cccc}
I_{2} & I_{2} & I_{2} & I_{2} \\
I_{2} & I_{2} & \varepsilon S_{1} & \varepsilon S_{2} \\
I_{2} & \varepsilon S_{1} & I_{2} & \varepsilon S_{3} \\
I_{2} & \varepsilon S_{2} & \varepsilon S_{3} & I_{2}
\end{array}\right)\right),
\end{gathered}
$$

where $\varepsilon= \pm 1$ and $S_{1}, S_{2}$ and $S_{3}$ are half-turns of equiangular coplanar axes.

However, the image by $\Lambda$ of a $\mathfrak{s}$-orbit of the first form described in Theorem 13 is the $\mathfrak{s}$-orbit of $p_{\pi / 2}$. As for $p_{0}$, its orbit is not exceptional. So no regular 
quadruple of the continuous family is rigid. Hence, among the regular direct equi-isoclinic quadruples which are listed in [3, Theorem 7], only one is rigid. The non-identity blocks of its Seidel matrix are all equal to a same rotation of angle $\pi / 2$.

\subsection{Real and complex $p$-tuples}

It is clear that any $p$-tuple of $G_{n}\left(\mathbb{R}^{p n}\right)$ can also be seen as a $p$-tuple of $G_{n}\left(\mathbb{C}^{p n}\right)$. Of course, there are, in general, many more $p$-tuples in $G_{n}\left(\mathbb{C}^{p n}\right)$. However, it is easy to derive from Theorem 3 the following

Corollary 19 Any special anisoclinic regular p-tuple of $G_{n}\left(\mathbb{C}^{p n}\right)$ actually belongs to $G_{n}\left(\mathbb{R}^{p n}\right)$.

Moreover, the following corollary is yields by the Theorems of sections 3.1.2 and 4.3

Corollary 20 Any common anisoclinic regular p-tuple of $G_{2}\left(\mathbb{C}^{2 p}\right)$ actually belongs to $G_{2}\left(\mathbb{R}^{2 p}\right)$.

From Proposition 18, we get the

Corollary 21 Any regular isoclinic triangle of $G_{n}\left(\mathbb{C}^{3 n}\right)$ actually belongs to $G_{n}\left(\mathbb{R}^{3 n}\right)$.

However, regularity in $G_{n}\left(\mathbb{C}^{p n}\right)$ does not imply realness with full generality, for there exist examples of regular isoclinic quadruples of $G_{2}\left(\mathbb{C}^{8}\right)$ which are not isometric to any quadruple of $G_{2}\left(\mathbb{R}^{8}\right)$. One of them is given by the following s-pair

$$
\left(\left[I_{2}\right]_{4},\left(\begin{array}{cccc}
I_{2} & I_{2} & I_{2} & I_{2} \\
I_{2} & I_{2} & R\left(\frac{\pi}{2}\right) & E_{1} \\
I_{2} & R\left(-\frac{\pi}{2}\right) & I_{2} & E_{2} \\
I_{2} & E_{1}^{*} & E_{2}^{*} & I_{2}
\end{array}\right)\right)
$$

where

$$
E_{1}=\frac{1}{4}\left(\begin{array}{cc}
-3 i & 2+i \sqrt{3} \\
-2+i \sqrt{3} & 3 i
\end{array}\right), E_{2}=\frac{1}{4}\left(\begin{array}{cc}
3 i & 2-i \sqrt{3} \\
-2-i \sqrt{3} & -3 i
\end{array}\right) .
$$

The reader can check that the formulae $(3), \ldots,(21)$ are satisfied for

$$
\begin{aligned}
& F_{1}=v_{\frac{\pi}{3}}\left(\frac{\pi}{2}, \frac{\pi}{2}\right) \\
& F_{3}=v_{\frac{\pi}{6}}\left(\frac{\pi}{2},-\frac{\pi}{2}\right) \\
& F_{4}=\frac{1}{4}\left(\begin{array}{cc}
-i \sqrt{3} & i-2 \sqrt{3} \\
i+2 \sqrt{3} & i \sqrt{3}
\end{array}\right) .
\end{aligned}
$$

Whether an anisoclinic regular $p$-tuple of $G_{n}\left(\mathbb{C}^{p n}\right)$ is necessarily isometric to a real one remains an open question. 


\subsection{Dimensions}

As we have seen, it is often difficult to determine the topology of the set of $\mathfrak{s}$-orbits for a given field $\mathbb{K}$ and given numbers $n, p$. It is of course easier to determine its dimension, especially in the anisoclinic real case. In this case, all the orbits are finite, whence the dimension of the set of $\mathfrak{s}$-orbits equals the dimension of the set of those matrices $U$ in $\mathfrak{s}$-pairs. On the other hand, since the left cosets are finite, $\operatorname{dim}(\operatorname{Im} \mathfrak{s})=\operatorname{dim}(O(n))=\frac{n(n-1)}{2}$. It follows that the space of anisoclinic $\mathfrak{s - o r b i t s}$ of $p$-tuples in $G_{n}\left(\mathbb{R}^{n p}\right)$ has dimension

$$
D_{\mathbb{R}}(n, p)=\frac{1}{2} n(n-1) p(p-2) \text {. }
$$

If we add the $\frac{1}{2} n p(p-1)$ critical angles, we obtain that a 'generic' (with a meaning which would deserve to be clarified) $p$-tuples of $G_{n}\left(\mathbb{R}^{n p}\right)$ is defined by $\frac{1}{2} n p(n p-2 n+1)$ numbers. This formula generalizes the one given at the end of [7], in the case $n=2$.

In the case of $G_{2}\left(\mathbb{C}^{2 p}\right), \operatorname{dim}(\operatorname{Im} \mathfrak{s})=\operatorname{dim} \mathbf{C}=2$ and the $\mathfrak{s}$-orbit of a generic $p$-tuple is always homeomorphic to $\mathbb{U}$, thus

$$
D_{\mathbb{C}}(2, p)=(p-2)(3 p-1)-1 .
$$

Hence a generic $p$-tuples of $G_{2}\left(\mathbb{C}^{2 p}\right)$ is defined by $4 p^{2}-8 p+1$ numerical invariants.

\section{References}

[1] M. Berger, Geometry I, Universitext, Springer-Verlag, Berlin, 1994.

[2] B. Et-Taoui and A. Fruchard, Sous-espaces equi-isoclins de l'espace euclidien, Adv. Geom. 9 (2009), 471-515.

[3] B. Et-Taoui and J. Rouyer, On p-tuples of equi-isoclinic 3-spaces in the Euclidean space, Indag. Mathem., N.S., to appear.

[4] A. Fruchard, Les triplets de Grassmann $G_{2}\left(\mathbb{R}^{6}\right)$, Geom. Dedicata 68 (1997), $123-144$.

[5] P. W. H. Lemmens and J. J. Seidel, Equi-isoclinic subspaces of Euclidean spaces, Nederl. Akad. Wet. Proc. Ser. A 76 (1973), 98-107.

[6] G. Masala, Regular triangles and isoclinic triangles in the Grassmann manifold $G_{2}\left(\mathbb{R}^{N}\right)$, Rend. Sem. Math. Univ. Torino, 57(2) (1999), 91-104.

[7] G. Masala, Conguence theorem for 4-tuples in the Grassmann manifold $G_{2}\left(\mathbb{R}^{8}\right)$, J. Geom. ,70 (2001) 117-132.

[8] J. R. Silvester, Determinants of Block Matrices, The Mathematical Gazette 84(501) (2000), 460-467 
[9] Y.-C. Wong, Differential geometry of Grassmann manifolds, Proc. Nat. Acad. Sci. USA 57 (1967), 589-594. 